\title{
The protective effect and mechanism of the FXR agonist obeticholic acid via targeting gut microbiota in non-alcoholic fatty liver disease
}

This article was published in the following Dove Press journal:

Drug Design, Development and Therapy

\author{
Dan-Ying Zhang ${ }^{1} * *$ \\ Lin $\mathrm{Zhu}^{2, *}$ \\ Hai-Ning Liu' ${ }^{1} *$ \\ Yu-Jen Tseng ${ }^{3}$ \\ Shu-Qiang Weng' \\ Tao-Tao Liu' \\ Ling Dong' \\ Xi-Zhong Shen ${ }^{1,4}$
}

'Department of Gastroenterology, Zhongshan Hospital of Fudan University, Shanghai 200032, People's Republic of China; ${ }^{2}$ Department of Geriatrics,

Zhongshan Hospital of Fudan University, Shanghai 200032, People's Republic of China; ${ }^{3}$ Department of Gastroenterology, Huashan Hospital of Fudan University, Shanghai 200040, People's Republic of China; ${ }^{4}$ Shanghai Institute of Liver Diseases, Zhongshan Hospital of Fudan University, Shanghai 200032, People's Republic of China

*These authors contributed equally to this work
Correspondence: Ling Dong; Xi-Zhong Shen

Zhongshan Hospital, Room 202, Building 3, I80 Fenglin Road Xuhui District, Shanghai, People's Republic of China Tel +862 1640419902070

Fax +862164432583

Email dong.ling@zs-hospital.sh.cn; xizhongshen@hotmail.com
Background: It is reported that various diseases such as non-alcoholic fatty liver disease (NAFLD) are associated with imbalance of microbiome. And FXR has been well investigated in liver diseases.

Purpose: The objective of this study was to identify the role of farnesoid X receptor agonist obeticholic acid via targeting gut microbiota in NAFLD.

Patients and methods: Male C57BL/6 mice were fed either a normal-chow diet or a high-fat diet (HFD). Obeticholic acid(30mg/( $\mathrm{kg} \cdot \mathrm{d}))$ and/or a combination of antibiotics were administered orally by gavage to mice for 12 weeks. Gut microbiota profiles were established through 16S rRNA amplicon sequencing. The effects of obeticholic acid on liver inflammation, the gut barrier, endotoxemia, gut microbiome and composition of the bile acid were also investigated.

Results: Obeticholic acid treatment can significantly improve obesity, circulation metabolism disorders, liver inflammation and fibrosis, and intestinal barrier damage caused by HFD. Removal of normal commensal bacteria can weaken the effect of obeticholic acid. The gut microbial structure was changed, and abundance of Blautia was increased significantly after treated with obeticholic acid. After obeticholic acid treatment, the concentration of taurinebound bile acid caused by HFD was reduced in the liver.

Conclusion: Taken together, these data suggest that obeticholic acid has aprotective effect on NAFLD via changing the components of gut microbiota, specifically increasing the abundance of Blautia.

Keywords: non-alcoholic fatty liver disease, farnesoid $\mathrm{X}$ receptor, gastrointestinal microbiome, bile acid, metabolic diseases

\section{Introduction}

Non-alcoholic fatty liver disease (NAFLD) is a serious liver disease with increasing prevalence due to the worldwide epidemic of obesity that has emerged as a major health problem. ${ }^{1}$ NAFLD or more specifically non-alcoholic steatohepatitis (NASH) is on the threshold of becoming, or has already become, the most common cause of liver disease. Prevention and treatment of NAFLD has gained the attention of many researchers. ${ }^{2}$ In recent years, new ideas for the treatment of this disease have been provided with the development of genetics and metabolism studies. ${ }^{3,4}$

Farnesoid X receptor (FXR) is a super family member of nuclear receptors. Activation by farnesol can regulate bile acid, lipids, cholesterol, and glucose homeostasis. ${ }^{5}$ People now realize that bile acids and their metabolites are the ligands of FXR and their main function in the human body is regulating the 
metabolism and enterohepatic circulation of bile acids. It has been suggested that the effects of the activation of FXR on various metabolic diseases, including fatty liver disease, type 2 diabetes, and obesity are beneficial. ${ }^{6}$ Obeticholic acid (INT-747, 6-ECDCA) is a potent FXR specific agonist that reduces liver fat and fibrosis in animal models of fatty liver diseases. ${ }^{7}$ It has been reported that there is a connection between liver health, fecal bile acid concentration and gut microbiota composition. $^{8}$

The composition and proportion of the gut microbiota in everyone is different from each other. The human microbiota is estimated contains $10^{14}$ bacterial cells, which is 10 times greater than the number of cells in the human body. ${ }^{9}$ The composition and function of the gut microbiota has been intensely studied in the past few years. ${ }^{10,11}$ The gut microbiota is important for human health. ${ }^{12}$ The gut microbiota is a central or a contributing cause of many diseases, such as obesity, inflammatory bowel disease, chronic periodontitis, irritable bowel syndrome, tropical enteropathy, and antibiotic-associated diarrhea and vaginosis. ${ }^{13-16}$ The human microbiome is an integral part of fundamental processes in the human body, such as digestion and absorption, energy homeostasis, angiogenesis, vitamin production, and maintenance of the intestinal barrier integrity. ${ }^{10}$ A western diet associated with weight gain can significantly alter the microbial composition. In recent years, high throughput techniques including 454 pyrosequencing of 16S rRNA genes can determine the composition of the gut microbiota rapidly. In our study, we detected gut microbiota using $16 \mathrm{~S}$ rRNA.

The pathophysiology of the relationship between bile acid metabolism and gut microbiota hasn't been well established. According the research reported, we speculate: obeticholic acid inhibites bile acid synthesis and promotes synthesis and excretion of bile salts through the FXR/FGF15 signaling pathway, and reduce the bile acid toxicity of hepatocytes, which can reduce the severity of NAFLD caused by high fat. On the other hand, obeticholic acid can improve intestinal mucosal barrier function, gut microbiota disorder and reduce intestinal endotoxemia and inflammation. Thence, the aim of our study was to explore the composition of bile acid and the change of gut microbiota in NAFLD mice model and to explore the protective effect and mechanism of the FXR agonist obeticholic acid by targeting the gut microbiota in NAFLD. We aimed to find a new potential therapeutic target for NAFLD.

\section{Materials and methods}

\section{Animals}

Forty-eight male C57BL/6 mice, aged 8 weeks, of SPF

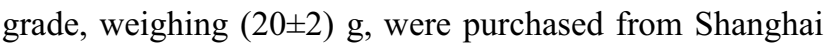
SLAC Laboratory Animals Co., Ltd. (Shanghai, China). The mice were housed in cages under a $12 \mathrm{~h}$ light-dark cycle and a temperature-controlled environment, starting with an adaptation period of 1 week and were fed normal chow. The high fat diet (Research diets D12492) and control diet were manufactured by Research Diets Inc. (New Brunswick, NJ, USA). The drinking water was tap water. All animal experiments used for this study were performed according to NIH standards as set forth in the "Guide for the Care and Use of Laboratory Animals" (DHHS pub. NIH 85-23 Rev. 1985). This study was approved by the Animal Ethics Committee of Zhongshan Hospital of Fudan University.

The 48 male C57BL/6 mice were randomly allocated into six groups with 8 mice in each group: control group with control diet for 24 weeks $(\mathrm{NC}, \mathrm{n}=8)$, group fed with HFD for 24 weeks (HF, $n=8$ ), group fed with the control diet and treated with obeticholic acid $(30 \mathrm{mg} /(\mathrm{kg} \cdot \mathrm{d}))$ dissolved in $1 \%$ methylcellulose after 12 weeks $(\mathrm{NI}, \mathrm{n}=8)$, group fed with the HFD and treated with obeticholic acid $(30 \mathrm{mg} /$ $(\mathrm{kg} \cdot \mathrm{d}))$ after 12 weeks (FI, $\mathrm{n}=8$ ), group fed with the control diet and treated with obeticholic acid $(30 \mathrm{mg} /(\mathrm{kg} \cdot \mathrm{d}))$ and a combination of antibiotics in drinking water after 12 weeks ( NV, $\mathrm{n}=8$ ), and a group fed with HFD and treated with obeticholic acid $(30 \mathrm{mg} /(\mathrm{kg} \cdot \mathrm{d}))$ and a combination of antibiotics after 12 weeks (FV, $\mathrm{n}=8)$. The combination of antibiotics included ampicillin (1 $\mathrm{g} / \mathrm{l})$, vancomycin $(500 \mathrm{mg} / \mathrm{l})$, neomycin sulfate $(1 \mathrm{~g} / \mathrm{l})$ and metronidazole $(1 \mathrm{~g} / \mathrm{l})$. Body weight and food intake were recorded on a weekly basis.

\section{Obesity portrait analysis}

Eight mice from each group were sacrificed at 24 weeks. The liver index was calculated as the liver weight (in grams)/body weight (in grams). The following serum biochemistry parameters were tested using a biochemical autoanalyzer (Hitachi 7170, Hitachi, Tokyo, Japan): alanine aminotransferase (ALT), aspartate aminotransferase (AST), alkaline phosphatase (ALP), $\gamma$-glutamyl transpeptidase (GGT), total bilirubin (TB) and glucose.

Lipopolysaccharide-binding protein (LBP) and adiponectin were assessed by a homogeneous fluorescence end-point assay kit (Hyglos $\mathrm{GmbH}$, Munich, Bavaria, Germany). 
Tumor necrosis factor- $\alpha$ (TNF- $\alpha$ ) levels were measured by enzyme-linked immunoassays with a commercial kit from Anogen (Mississauga, Ontario, Canada).

Serum triglyceride, cholesterol, low-density lipoproteincholesterol (LDL-C), and high-density lipoprotein-cholesterol (HDL-C) were measured enzymatically. Liver tissues were stored at $-80^{\circ} \mathrm{C}$, and the tissues were pulverized under liquid nitrogen and lyophilized. The detail experimental methods referred to the kit protocol. The triglycerides, cholesterol, LDL-C, and HDL-C levels in the liver were analyzed.

\section{Histopathological examination of liver and intestine tissue}

The liver, ileum and colon tissues were fixed with $10 \%$ formalin, embedded in paraffin, and stained with hematoxylin-eosin. After routine processing, paraffin sections were cut into $5 \mu \mathrm{m}$ thickness slices and stained with hematoxylin-eosin (HE). Oil red O, and Masson's trichrome (Sigma-Aldrich Corp., St. Louis, MO, USA) was performed with cryosection .Evaluation was performed under a light microscope. The structural changes of cells and tissues were observed.

\section{Real-time quantitative PCR analysis}

To assess the level of gene expression of tight junction protein1 (ZO-1), occludin, TNF- $\alpha$, interleukin-1 $\beta$ (IL-1 $\beta$ ), IL-10, fibroblast growth factor 15 (Fgf15), cholesterol $7 \alpha$ hydroxylase (CYP7A1), sterol 12a-hydroxylase (CYP8B1), small heterodimer partner (SHP) and bile salt export pump (BSEP), the liver, ileum and colon tissues of the animals were collected. Total RNA was extracted from $20 \mathrm{mg}$ of tissue using an RNA Purification Kit (Takara Bio, Inc., Otsu, Shiga, Japan) according to the manufacturer's protocol. RNA concentration was measured with the NanoDropND1000 (NanoDrop, Wilmington, DE, USA). cDNA was generated from total RNA using a Reverse Transcriptase Kit (Takara Bio, Inc., Otsu, Shiga, Japan). Then, $1 \mathrm{mg}$ of RNA was used for measuring target gene expression by reverse transcription quantitative real-time PCR (RT-qPCR). The forward and reverse primers of the target genes are listed in Table S1. The RT-qPCR reaction was performed using SYBR premix Ex Taq (Takara Bio, Inc., Otsu, Shiga, Japan). The reaction mixture was incubated for $300 \mathrm{sec}$ at $95^{\circ} \mathrm{C}$, followed by 35 amplification cycles of $30 \mathrm{sec}$ at $95{ }^{\circ} \mathrm{C}, 30 \mathrm{sec}$ at $60^{\circ} \mathrm{C}$, and $30 \mathrm{sec}$ at $72{ }^{\circ} \mathrm{C}$, on an ABI PRISM 7,500 Sequence Detection System (Applied Biosystems, Foster City, CA, USA). Cycle threshold $(\mathrm{Ct})$ values of all samples were normalized using the $2^{-\Delta \Delta \mathrm{Ct}}$ method. Each qPCR assay was performed in triplicate.

\section{Western blotting}

The proteins were transferred to nitrocellulose membranes (Thermo Fisher Scientific China, Beijing, China). The membranes were blocked in 5\% nonfat milk in Trisbuffered saline (TBS) with Tween-20 detergent for $2 \mathrm{~h}$ at room temperature, incubated with primary antibodies (mice anti-occludin, ZO-1, 1:1,000 and anti-GAPDH, 1:5,000) overnight at $4{ }^{\circ} \mathrm{C}$ and then incubated with secondary antibodies for $2 \mathrm{~h}$ at room temperature. The chemiluminescent signals of the bands were detected using the ChemiDoc MP system (Bio-Rad, Hercules, CA, USA).

\section{Microbial diversity analysis}

The same operational methods as in our previous study were used. ${ }^{17}$ Linear discriminant analysis (LDA) coupled with effect size (LEfSe) measurements, which are based on the Kruskal-Wallis sum-rank test and Wilcoxon rank-sum test, were used to identify taxa significantly different (biomarkers) among groups, with an LDA score threshold of 3 and $P<0.05$.

\section{Analysis of bile acids}

Samples (10 mg each) of liver, gallbladder, ileum, cecum, and feces in each group were collected. The samples were placed in a $1.5 \mathrm{ml}$ grinding tube with magnetic beads. Methanol/ water (1/1) $200 \mu \mathrm{l}$ was added along with $0.10 \mu \mathrm{mol} / 1$ of LCAD4, CA-D4 and UDCA-D4 (C/D/N Isotopes, Pointe-Claire, Quebec, Canada) for use as the internal standard. Ultra-highperformance liquid chromatography-mass spectrometry (UPLC-MS)/MS was used to detect total bile acid profiles. Detailed methods were described in a prior study. ${ }^{18}$

\section{Statistical analysis}

Values are expressed as the mean \pm SD. SPSS 19.0 (IBM Corporation, Armonk, NY, USA) was used for the analysis of variances. Statistical analysis was performed by oneway ANOVA or Kruskal-Wallis tests followed by Bonferroni correction for parametric and nonparametric analyses, respectively. Differences were considered statistically significant at $P<0.05$.

\section{Results \\ Obeticholic acid improves obesity portraits in HFD-fed mice}

The body weight and liver index of mice in the HF group were higher than that of the NC group significantly $(P<0.05)$ (Figure 1A, B and D). Those of the FI group were significantly lower than in the HF group $(P<0.05)$. Obeticholic 

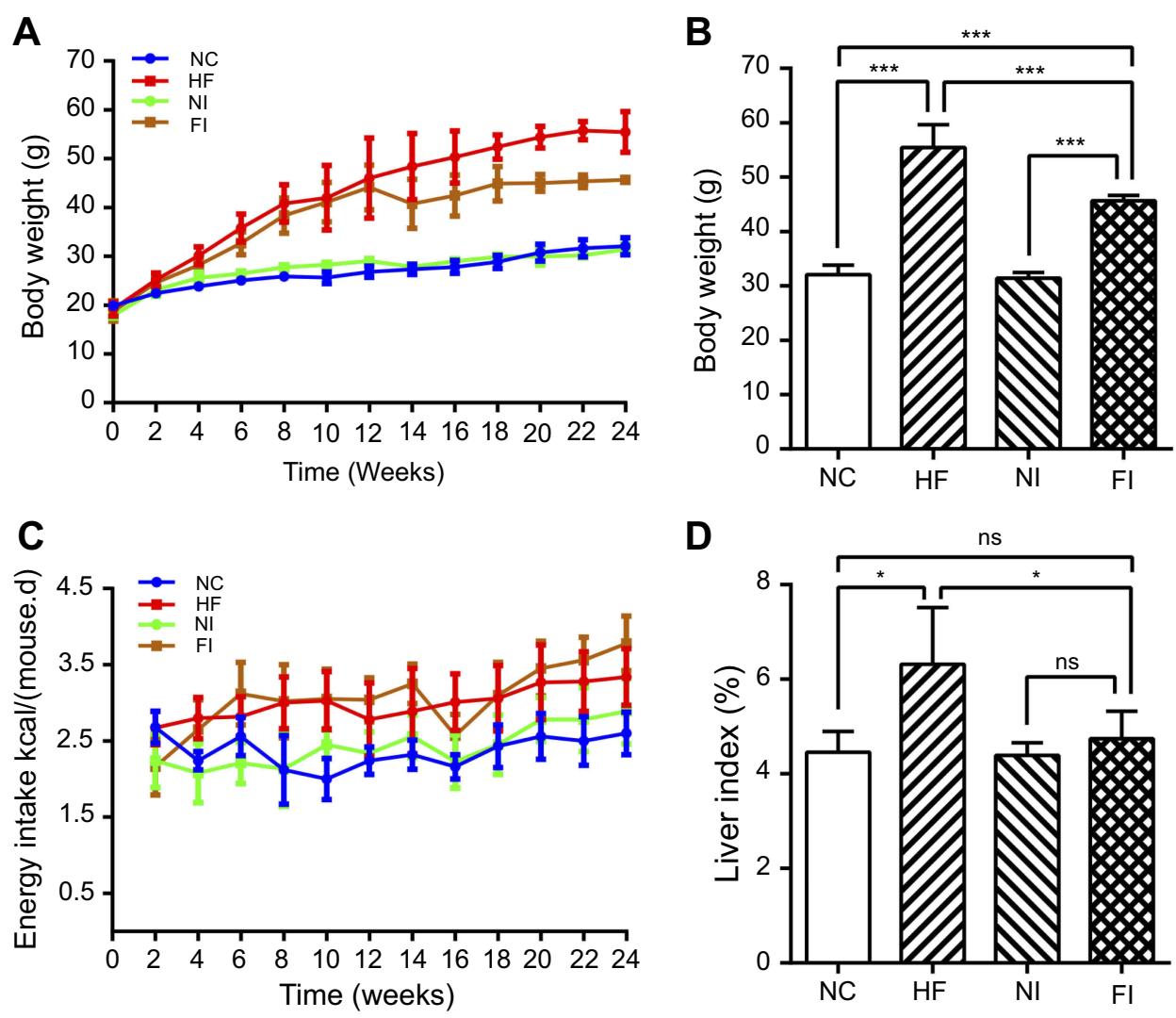
Figure I Obesity portraits. (A) Body weight through the 24 -week period. (B) Body weight in the 24 th week. (C) Energy intake through the 24 -week period. (D) Liver
index. $* P<0.05 ; * * * P<0.00 \mathrm{I}$.
Abbreviations: $\mathrm{NC}$, control group fed with control diet; $\mathrm{HF}$, group fed with high fat diet; $\mathrm{Nl}$, group fed with the control diet and treated with obeticholic acid $(30 \mathrm{mg} /$
$(\mathrm{kg} \cdot \mathrm{d}))$; $\mathrm{Fl}$, group fed with the high fat diet and treated with obeticholic acid $(30 \mathrm{mg} /(\mathrm{kg} \cdot \mathrm{d})) ; \mathrm{ns}$, not statistically significant.

acid could reduce the body weight and liver index in fat mice induced by a western high-fat diet (HFD). The energy intake of mice on the HFD (including the HF and FI group) were greater than that of the NC and NI group (Figure 1C). The energy and food intake of the mice in the FI group were similar to that of the HF group. However, the weight gain and liver index of mice in the FI group were lower than that of the HF group at the end of the experiment. This indicated that germ-free animals require a significantly higher caloric intake to maintain the same body weight.

Serum levels of ALT, AST, GGT, TB, LBP, TNF- $\alpha$, glucose, LDL-C, liver cholesterol, triglycerides and HDL-C were substantially elevated by HFD feeding $(P<0.05)$ (Figures $\mathrm{S} 1$ and $\mathrm{S} 2)$. The results showed that obeticholic acid treatment resulted in significant reductions in the above indexes $(P<0.05)$. Through analyses of hepatic function, obeticholic acid treatment was shown to reduce liver damage, inflammation and metabolic disorders and lipid metabolism disorders caused by HFD.

\section{Obeticholic acid improves liver inflammation and fibrosis}

HFD has been shown to cause liver inflammation, even fibrosis and cirrhosis. Figure 2 shows that the liver in the HF group has severe steatosis and mixed vacuolar degeneration and that the steatosis and inflammation can be reduced after obeticholic acid treatment. The histological examination of the liver sections by oil red O staining and Masson's trichrome confirmed the beneficial effects of obeticholic acid treatment on hepatic fibrosis and fat deposition.

\section{Obeticholic acid protects intestinal barrier function}

Figure 2 show that the mucosal layer, submucosal layer and muscular layer of both the colon and ileum in the NC and NI group had no obvious inflammation. The severity of inflammation in mucosa and submucosal layer in the HF group was mild or moderate. Obeticholic acid treatment can improve intestinal inflammation. 

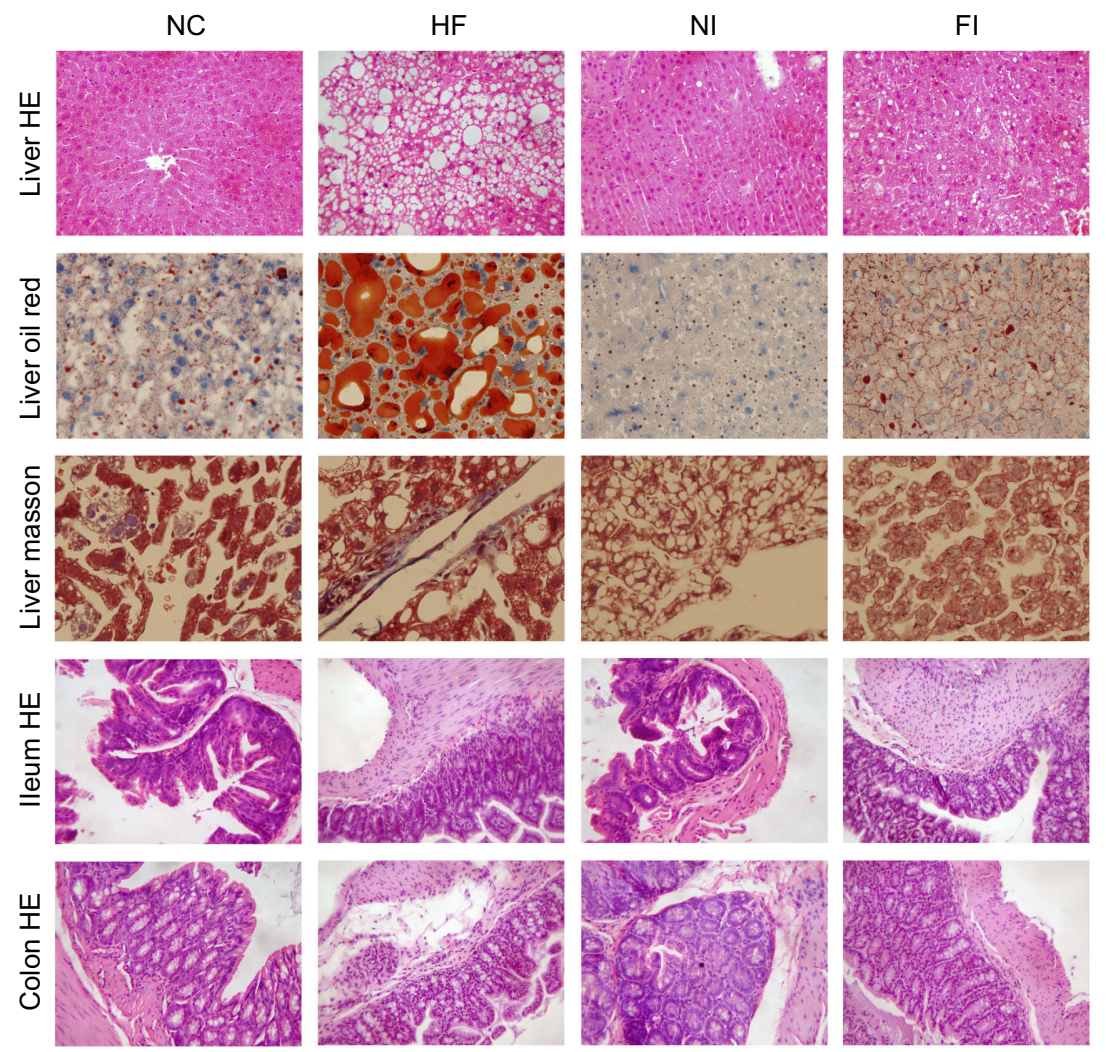

Figure 2 Histopathological staining of liver, ileum and colon tissues. Representative liver HE, oil red O and Masson's trichrome staining, and HE staining of ileum and colon tissues (200x magnification).

Abbreviation: HE, hematoxylin-eosin.

The ZO-1 and occludin were both downregulated at the mRNA level in the HF group (Figure 3A, B, $\mathrm{G}$ and $\mathrm{H}$ ) while $\mathrm{ZO}-1$ and occludin were upregulated at the mRNA level in the FI group. The results suggested that HFD can cause damage to the colon and ileum mucosal barrier, while obeticholic acid treatment can repair the damage. At the protein level, the change trend of ZO-1 and occludin was consistent with the mRNA levels (Figure 3F and L).

In the ileum and colon tissues, the inflammatory factor TNF- $\alpha$ and IL-1 $\beta$ expression in the HF group were upregulated, exceeding the level of significance, whereas the expression of the anti-inflammatory factor IL-10 was downregulated (Figure 3C-E and I-K). The FI group displayed significant opposite results compared with the HF group in the colon and ileum. The results showed that HFD can induce a release of inflammatory factors and reduce the release of inflammatory protective factors. Obeticholic acid treatment can relieve the damage to the intestinal mucosal barrier secondary to HFD.

\section{Effects of obeticholic acid treatment interventions on gut microbiota}

A total of $8,59,109 \times 2$ reads were obtained for 32 samples. The Shannon index of NC, HF, NI and FI were 3.35, 2.67, 3.52 and 3.83, respectively. Shannon-Wiener analysis showed the bacterial diversity of the HF group was significantly decreased compared with the other groups. The results of Chao1 and the abundance-based coverage estimator (ACE) index showed the same trend as the Shannon index.

The overall structural changes of the gut microbiota were analyzed using multivariate statistical methods. Principal coordinate analysis (PCoA) score plots showed that HFD feeding resulted in a greater structural shift along the first principal component (PC1). The treatment with obeticholic acid significantly reverted the HFD-induced variations along the PC1 (Figure 4A). The nonmetric multidimensional scaling (NMDS) analysis based on the Bray-Curtis distance also confirmed the bacterial communities in the four groups were different from each other (Figure 4B). 

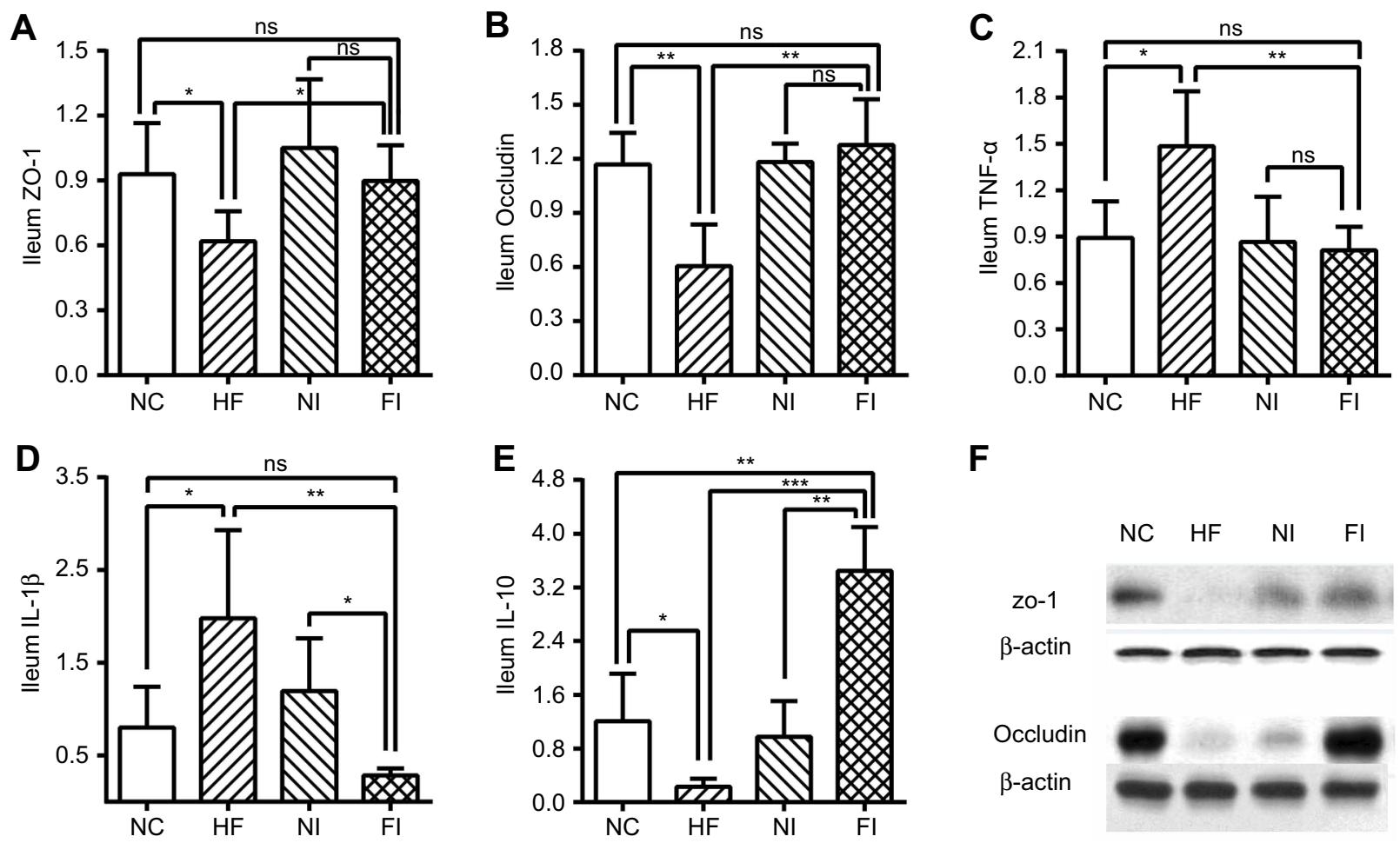

$\mathbf{F}$
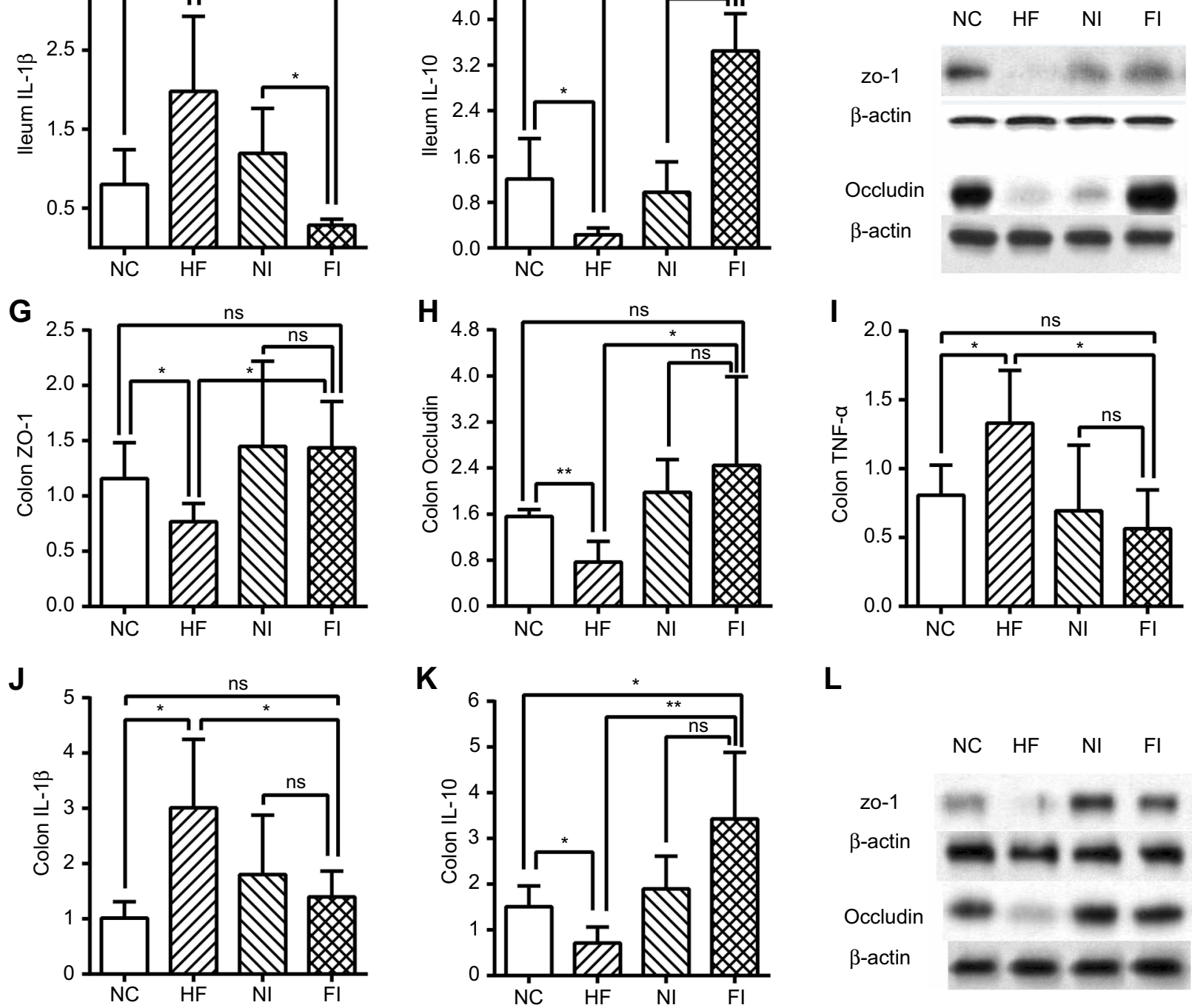

$\mathbf{L}$

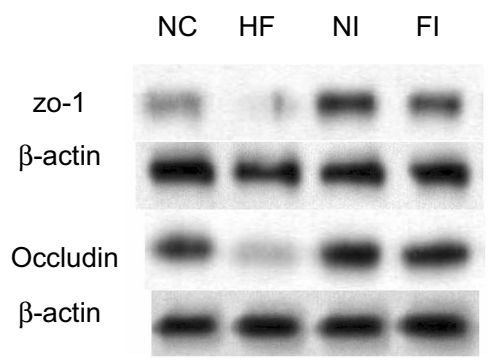

Figure 3 Intestinal barrier function. (A) Relative mRNA expression of ZO-I, (B) occludin, (C) TNF- $\alpha$, (D) IL-I $\boldsymbol{\beta}$ and (E) IL-I0 in ileum tissue. (F) Protein expression of ZO-I and occludin in ileum tissue. (G) Relative mRNA expression of ZO-I, (H) occludin, (I) TNF- $\alpha$, (J) IL-I $\boldsymbol{\beta}$ and (K) IL-I0 in colon tissue. (L) Protein expression of ZO-I and occludin in colon tissue. $* P<0.05 ; * * P<0.01 ; * * * P<0.001$; ns, not statistically significant.

Abbreviations: ZO-I, tight junction protein-I; TNF- $\alpha$, tumor necrosis factor- $\alpha$; IL, interleukin.

Figure $4 \mathrm{C}$ and $\mathrm{D}$ show the bacterial composition of the different communities at the phylum and genus level. The results showed that the most abundant microbiota in the
HF group was the Bacteroidetes, while the most abundant microbiota in the NI and FI groups was Firmicutes at the phylum level. Obeticholic acid increased the abundance of 


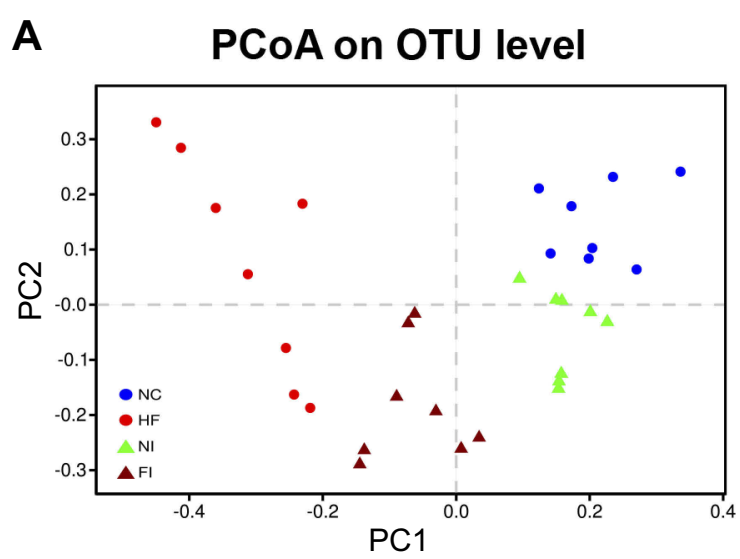

\section{Community barplot analysis}

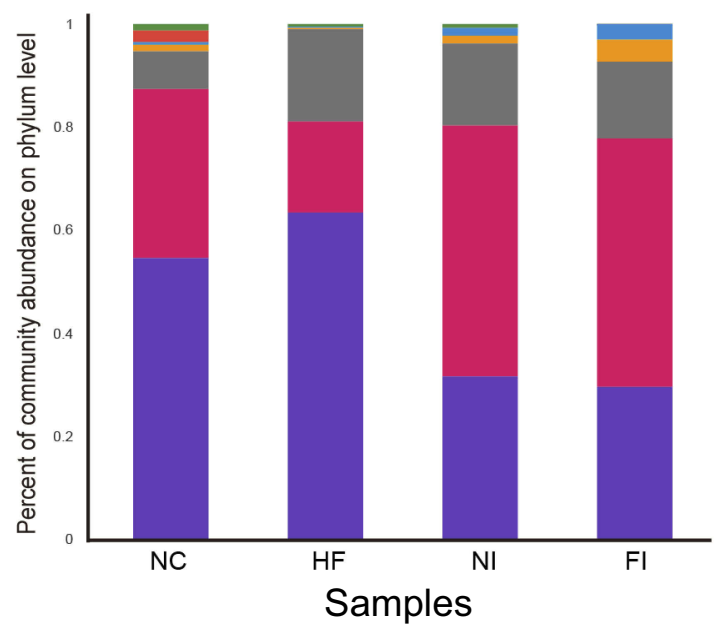

Verrucomicrobia Deferribacteres

Actinobacteria

Proteobacteria Firmicutes

Bacteroidetes Others

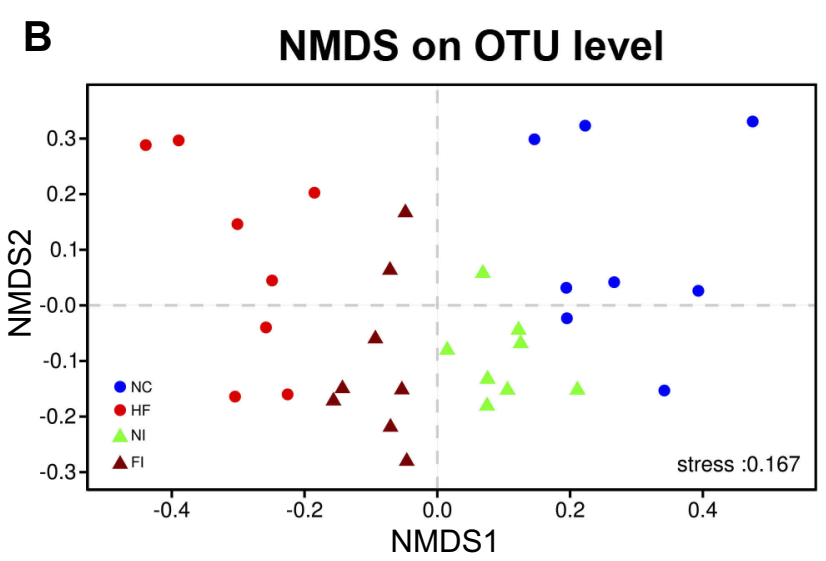

D
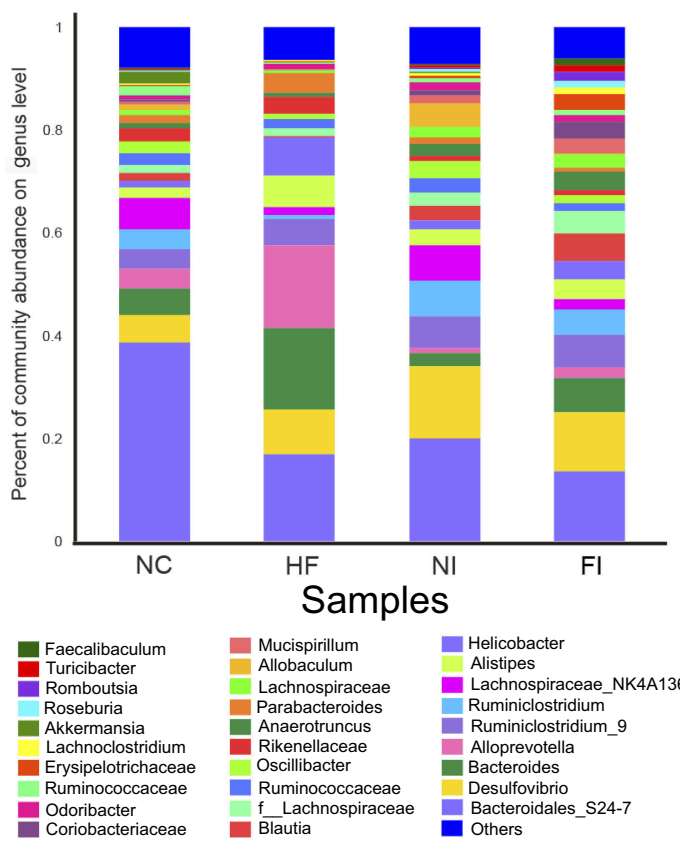

Helicobacter Alistipes Lachnospiraceae_NK4A136

Figure 4 Score plots of multivariate analysis on OTU level and community barplots. (A) PCoA and (B) NMDS on OTU level. (C) Community barplot on the phylum and (D) genus level.
Abbreviations: PCoA, principal coordinate analysis; OTU, operational taxonomic unit; NMDS, nonmetric multidimensional scaling; ZO-I, tight junction protein- I; TNF- $\alpha$, tumor necrosis factor- $\alpha$; IL, interleukin..

Firmicutes and decreased the relative abundance of Bacteroidetes significantly, while no obvious change was observed in the HF and FI groups. The content of Firmicutes was significantly increased after obeticholic acid treatment in both the control and high-fat group. The relative abundance of Proteobacteria and Bacteroidetes was increased, while Firmicutes was decreased in the high-fat group compared with the control group. At the genus level, obeticholic acid showed enriching effects on Desulfovibrio, Blautia, Mucispirillum, Ruminiclostridium, and Anaerotruncus, and inhibiting effects on Bacteroides, and Parabacteroides in both the NI and FI group (Figure 5A).

LEfSe and LDA were conducted to better understand the differences of gut microbiota at the genus level (Figure 5B and C). HFD increased the proportion of Bacteroides, Helicobacter and Alloprevotella. The analysis of LEfSe showed the difference was significant. The FI group showed enriching effects on Blautia and Mucispirillum and inhibiting effects on Bacteroides and Parabacteroides in both the NI and FI groups. The detailed microbiota is shown in Table S2. 
A One-way ANOVA bar plot

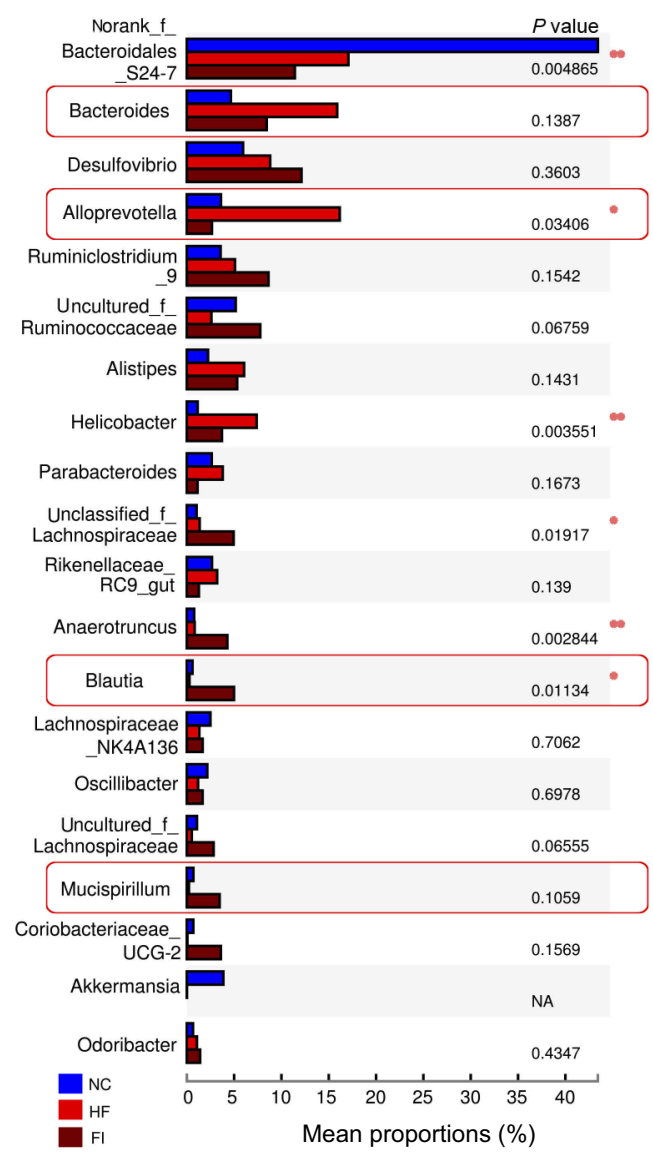

B

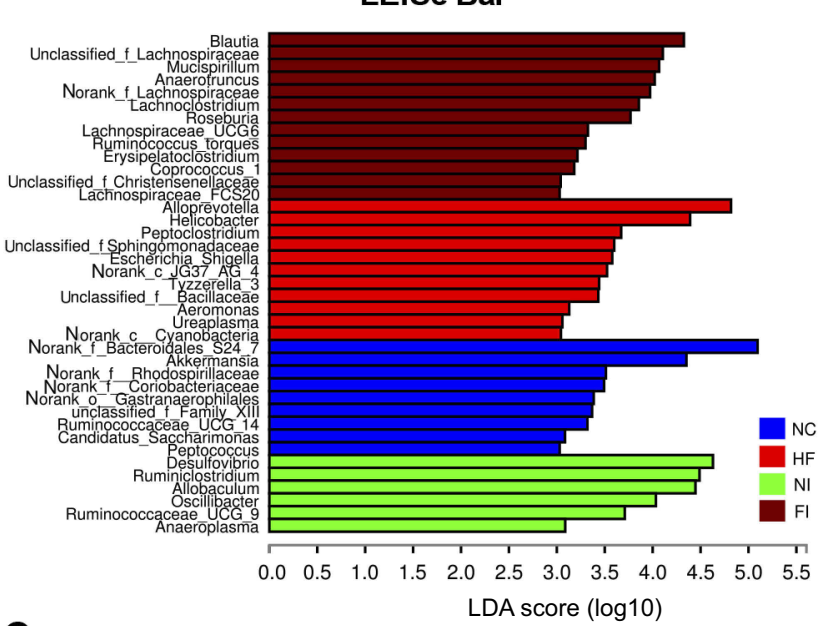

C

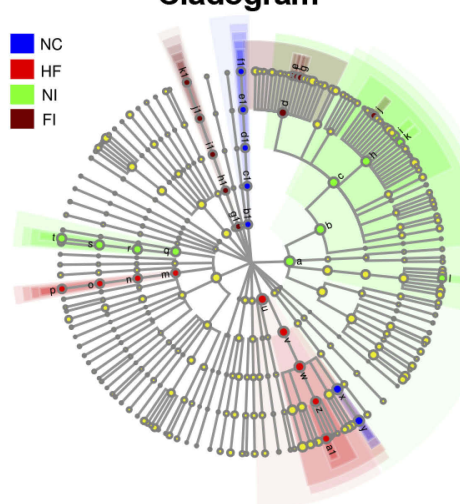

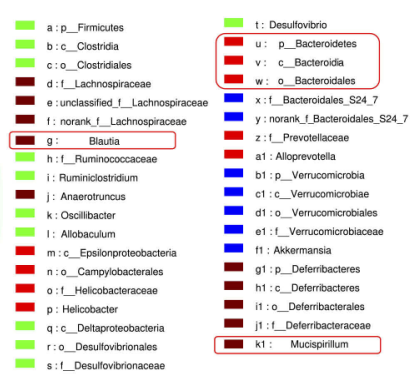

Figure 5 Difference analysis of gut microbiota. (A) One-way ANOVA bar plot of the top 20 genera with the most abundant expression. (B) LDA scores of taxa enriched on the genus level. Only taxa with an LDA significant threshold $>3$ are shown. (C) Taxonomic cladogram generated by LEfSe analysis from phylum to genus level. $* P<0.05$, $* * P<0.01$. Abbreviations: ANOVA, analysis of variance; LEfSe, linear discriminant analysis coupled with effect size; LDA, linear discriminant analysis.

\section{Obeticholic acid influences the composition of the bile acid}

The results showed that the bile acid content in the gallbladder was the highest, followed by the terminal ileum. In the liver, gallbladder, and terminal ileum, the total bile acid content of the NAFLD model group was significantly increased, while the obeticholic acid intervention group had a reduced bile acid content in the liver, gallbladder, and terminal ileum. There was no significance difference in the bile acid in the cecum and ileum among these groups. The composition of bile acid in each group revealed a high concentration of taurine-bound bile acid in the liver and gallbladder of the normal control mice, while the taurine-bound bile acid in the high-fat group was also increased. After obeticholic acid treatment, the tissue was dominated by unconjugated bile acids. In feces, the bile acid component ratio was significantly altered similar to the tissues, while the obeticholic acid group was similar to the control group (Figure S3).
Analysis of individual bile acids revealed that taurocholic acid (TCA) was the most common bile acid in the gallbladder of the HF group of mice (Figure 6). TCA and tauro- $\alpha$ muricholic acid (TaMCA) contents were decreased after obeticholic acid treatment, while chenodeoxycholic acid (CDCA) and $\beta$-muricholic acid $(\beta \mathrm{MCA})$ contents were increased in the FI group. In the distal ileum tissue, TaMCA, tauro- $\beta$ muricholic acid (TRMCA) and TCA were the most abundant bile acids. Obeticholic acid can increase the content of taurochenodeoxycholic acid (TCDCA), T $\alpha \mathrm{MCA}$, and T $\beta \mathrm{MCA}$ in the distal ileum of obese mice. In the liver, TCA was the most abundant in the HF group. Obeticholic acid intervention can reduce the TCA content. In the cecum, deoxycholic acid (DCA), muricholic acid (MCA), and $\beta \mathrm{MCA}$ were abundant. Obeticholic acid intervention can reduce the content of DCA, UDCA, LCA, $\alpha$-muricholic acid ( $\alpha$ MCA), $\beta$ MCA, and CA. In feces, compared with the NC group, the content of DCA and TCA in the HF group were significantly increased while the 

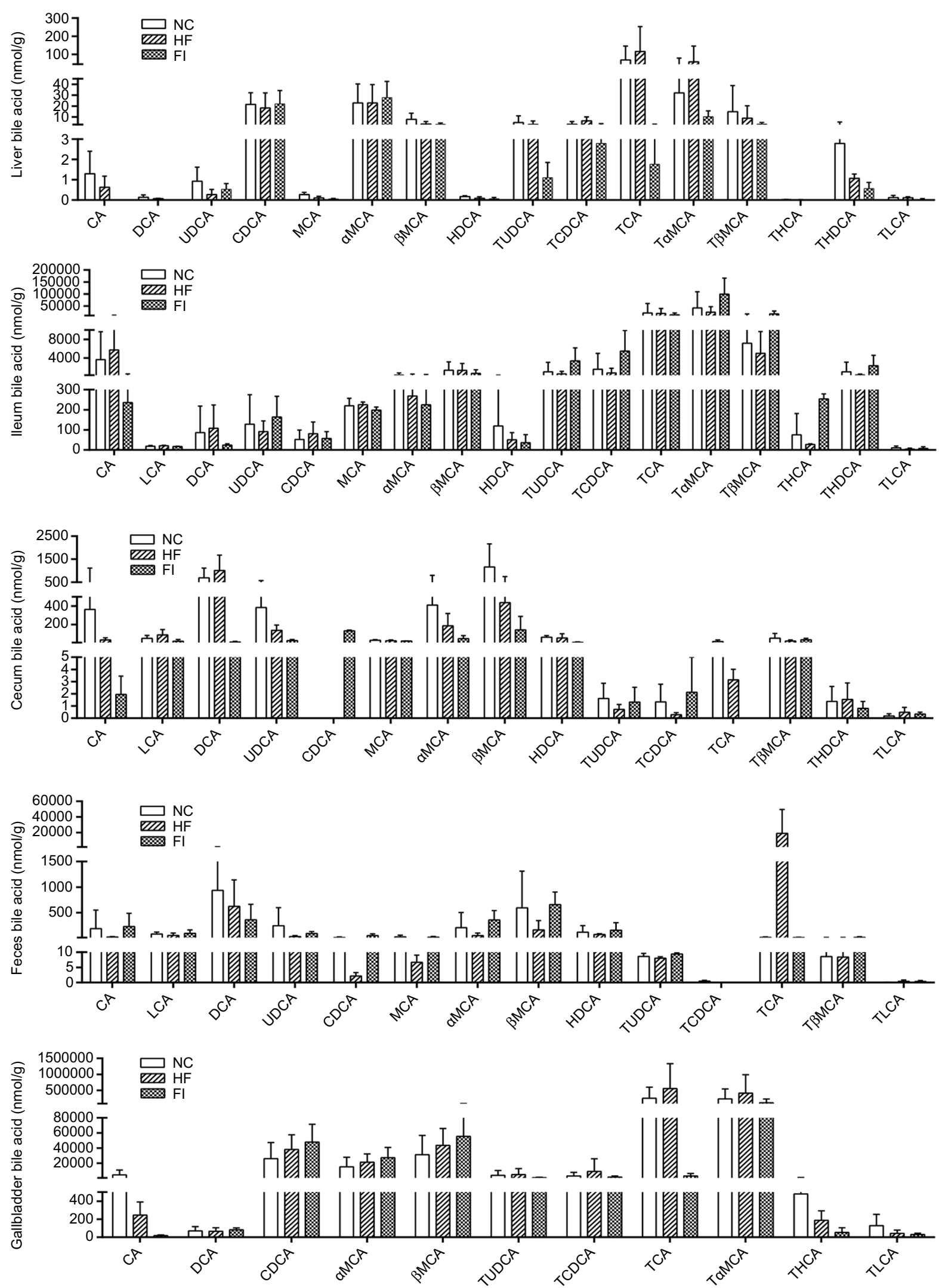

Figure 6 Bile acid levels in liver, ileum, cecum, feces and gallbladder.

Abbreviations: CA, cholic acid; LCA, lithocholic acid; DCA, deoxycholic acid; UDCA, ursodeoxycholic acid; CDCA, chenodeoxycholic acid; MCA, muricholic acid; $\alpha$ MCA, $\alpha$-muricholic acid; $\beta$ MCA, $\beta$-muricholic acid; HDCA, hyodeoxycholic acid; TUDCA, tauro-ursodeoxycholic acid; TCDCA, tauro-chenodeoxycholic acid; TCA, tauro-cholic acid; T $\alpha$ MCA, tauro- $\alpha$-muricholic acid; T $\beta$ MCA, tauro- $\beta$-muricholic acid; THDCA, tauro-hyodeoxycholic acid; TLCA, tauro-lithocholic acid. 


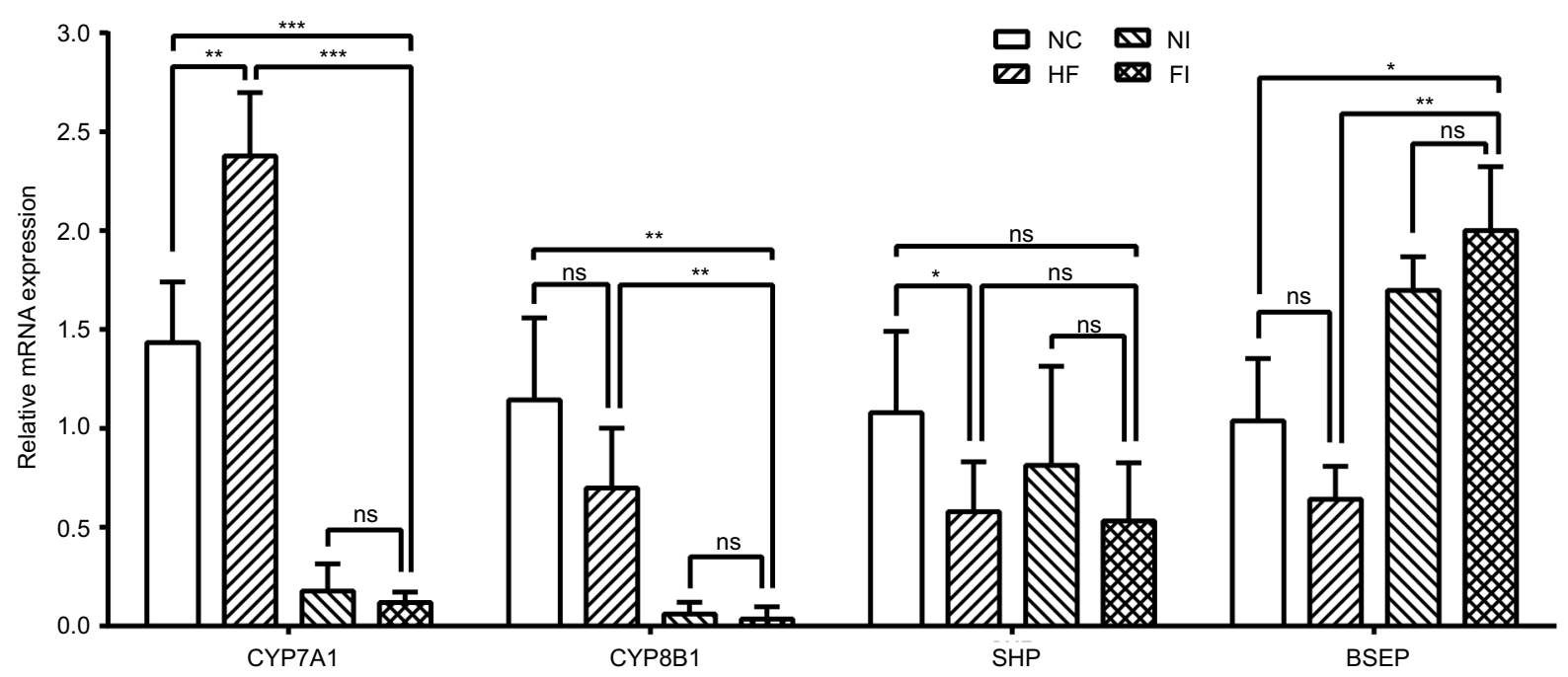

Figure 7 mRNA expression of bile acid metabolism. mRNA expression of CYP7AI, CYP8BI, SHP and BSEP. $* P<0.05 ; * * P<0.01$; $* * * P<0.00 I$; ns, not statistically significant. Abbreviations: CYP7AI, hepatic cholesterol $7 \alpha$-hydroxylase; CYP8BI, sterol I $2 \alpha$-hydroxylase; SHP, small heterodimer partner; BSEP, bile salt export pump.

DCA and TCA levels in the FI group were similar to the NC group. CA, MCA, $\alpha \mathrm{MCA}$ and $\beta \mathrm{MCA}$ were increased in the FI group compared with the HF group (Table S3).

\section{Obeticholic acid alters the expression} gene profile involved in bile acid synthesis, conjugation, and reabsorption

Figure 7 shows that treatment of high-fat mice with the FXR agonist obeticholic acid suppressed CYP7A1 and CYP8B1 expression in the liver. It can also significantly increase the expression of BSEP in the liver. In addition, Fgf15 expression in the HF group in the ileum and colon was more than twice than that of the NC group $(P<0.05)$, whereas Fgf15 expression in the NI and FI groups were higher than that of the HF group $(P<0.05)$ significantly. Thus, these results demonstrate that reduced FXR signaling in HF mice can be reversed by treatment with a specific FXR agonist.

\section{Removal of normal commensal bacteria can weaken the effect of obeticholic acid} We showed that the serum ALT, AST, GGT, TB, glucose, LBP, TNF- $\alpha$ and LDL-C, and liver triglyceride and cholesterol levels of the FV group increased significantly compared with those of the FI and NV group (Figure S4). HE and oil red $\mathrm{O}$ staining showed that liver steatosis and inflammation in the FV group were more severe than in the FI and NV groups (Figure S5). This finding indicates that obeticholic acid plays a variety of roles via targeting gut microbiota. The effect of obeticholic acid on liver inflammation and fibrosis and intestinal barrier function was weakened after removing normal intestinal commensal bacteria by broad-spectrum antibiotics.

\section{Discussion}

Because of the modern sedentary and food-abundant lifestyle, NAFLD is one of the leading causes of chronic liver diseases now. ${ }^{19,20}$ It is widely acknowledged that it encompasses a spectrum of disease states, from steatosis (fatty liver) to NASH followed by progression to fibrosis, cirrhosis and hepatocellular carcinoma. ${ }^{21}$ In our study, C57BL/6 widetype mice fed with HFD had significant hepatic triglyceride and cholesterol accumulation, increased serum levels of ALT, AST, GGT, TB, and exhibited histological features of $\mathrm{NASH}$, including mixed steatosis and lobular inflammation, as our results show in Figure 2. HFD also increased serum LBP concentrations and the TNF- $\alpha$ level.

FXR is a nuclear receptor displaying a key role in NAFLD and NASH pathogenesis. FXR possesses anti-inflammatory properties by antagonizing NF- $\mathrm{kB}$ signaling. ${ }^{22}$ FXR regulates bile acid synthesis through two distinct mechanisms. In liver, FXR upregulates the expression of SHP. SHP can repress the transcription of bile acid synthesis enzymes, cytochrome $\mathrm{P} 450$ 7A1 and 8B1 (CYP7A1 and CYP8B1), decreasing bile acid synthesis. ${ }^{23}$

Obeticholic acid is a novel derivative of cholic acid, which acts as a potent and selective FXR agonist displaying anticholeretic activity. It has been approved for the treatment of primary biliary cholangitis (PBC). The 
interim results from the National Institutes of Health (NIH)-sponsored FLINT clinical trial revealed that obeticholic acid is effective for liver damage due to NASH. ${ }^{24}$ It has also been proven to improve hepatic steatosis, fibrosis and portal hypertension. ${ }^{25-28}$ In our study, obeticholic acid with HFD prevented hepatic triglyceride and cholesterol accumulation, altered histological features of hepatic steatosis and lowered the serum level of the liver functional enzymes ALT, AST, GGT and TB

LBP plays a crucial role in response to Gram-negative bacteria infection and modulates cellular signals from lipopolysaccharide (LPS). ${ }^{29}$ Studies have shown that LPS can cause inflammation, leading to immune activation and release of cytokines. ${ }^{30,31}$ However, it has a short half-life. LBP is considered more reliable than LPS in serum due to its long half-life. ${ }^{32}$ It has been reported that serum LBP concentrations are higher in individuals who are overweight, obese or have type 2 diabetes. ${ }^{33}$ In our study, the HF group mice exhibited reduced the expression of tight junction protein (ZO-1 and occludin) in ileum and colon. It can increase intestinal permeability and cause serious LPS/ LBP-related metabolic endotoxemia, which is consistent with our study results. Adiponectin is an adipokine that is abundantly produced by adipose tissue. It has antidiabetic, anti-atherogenic and anti-inflammatory effects. ${ }^{34}$ Adiponectin is important for energy metabolism. It can regulate glucose, lipid metabolism and increase insulin sensitivity. Although the present research did not establish a significant difference in adiponectin among groups, a difference in glucose lipid metabolism and endotoxemia was observed.

The difference of gut microbiota is closely related to NAFLD. In our study, it is investigated the antiinflammatory effects of obeticholic acid on NAFLD. We also explored the possibility these effects are caused by gut microbiota. The gut microbiota plays an effective role within the gut, and it acts in other parts of the enterohepatic system too, such as regulating bile acid synthesis in the liver. $^{35}$ In our study, Shannon-Wiener analysis showed that the bacterial diversity of the HF group was significantly decreased, while obeticholic acid made the decline in bacterial diversity reverse. A potential mechanism for this could be that obeticholic acid can improve liver cholestasis, intestinal permeability and improve the intestinal flora imbalance caused by HFD. It can reduce the level of LBP and exogenous pathogenic antigens. The result is reinforced by our finding. Increased endotoxemia and inflammation in humans is a key to the development of NAFLD. ${ }^{31}$
LEfSe was used to identify potential discriminating taxa between these groups. The results showed that the composition of gut microbiota of HFD group is different from that of control group. We found that Blautia and Mucispirillum abundance were significantly increased in HFD mice intervened by obeticholic acid. Blautia is a member of the Firmicutes, which can produce shortchain fatty acids (SCFAs) and in turn maintains the integrity of the intestinal mucosal barrier. Blautia is one of a predominant member of a larger group of bacteria. And it mediates beneficial anti-inflammatory effects and disappear as a consortium. ${ }^{36}$ SCFAs have diverse physiological roles in body functions. ${ }^{37}$ SCFAs contribute to shaping of the gut environment, influencing the physiology of the colon, and can be utilized as energy sources by host cells and intestinal microbiota, while also participating in different host-signaling mechanisms. ${ }^{38}$ It is showed that SCFAs, including butyrate contribute towards maintaining epithelial integrity, hormone secretion, gut motility, and reducing appetite and inflammation, ${ }^{39,40}$ all of which are associated with NAFLD. ${ }^{41}$

To investigate whether obeticholic acid works via targeting gut microbiota in NAFLD, we compared the therapeutic effect between the FV and FI group. The results showed that although the same dose of obeticholic acid was given to the same high-fat diet mice, the therapeutic effect, including reducing obesity, liver function damage, endotoxemia, glycolipid metabolism, liver inflammation and fibrosis, and intestinal barrier damage, was weakened in the FV group. Therefore, obeticholic acid plays an important role in NAFLD via targeting gut microbiota. In future research, fecal microbiota transplantation (FMT) from donor rats treated with obeticholic acid to HFD-fed receptor rats may show a similar treatment efficacy. This can further establish the interaction between obeticholic acid and gut microbiota. If a distinctive "target microbiome" can be identified, treatment with selective probiotics may result in similar treatment efficacy as obeticholic acid. However, the composition of the gut microbiota is rather complex and warrants further investigation.

Similar to bilirubin, bile acids are a major component of the marked increase in cholestasis. In our study, bile acids in tissues of HFD mice increased significantly, which indicated that cholestasis was present in the HF group, while obeticholic acid treatment can improve cholestasis. Dupont et $\mathrm{al}^{42}$ reported that taurine-bound bile acids are the dominant bile acid in the normal rat liver, which is consistent with our study results. The present study showed taurine- 
bound bile acids were increased in the liver and gallbladder tissue after HFD. After obeticholic acid intervention, the concentration of taurine-bound bile acid caused by HFD was reduced. We found obeticholic acid increased intestinal Fgf15 expression and suppressed hepatic CYP7A1 and CYP8B1 expression, which is consistent with a previous literature report. $^{43}$

\section{Conclusion}

Our research adds to accumulating evidence that obeticholic acid can prevent metabolic disorders including NASH induced by HFD. The results of our study support the hypothesis that structural changes of gut microbiota contributed by obeticholic acid can improve liver inflammation and cholestasis. Obeticholic acid treatment specifically increased the abundance of Blautia; this finding suggests that the beneficial effect of obeticholic acid is partly attributable to the associated increase in Blautia. The present study data raise the potential application of pharmacological manipulation of gut microbiota as a treatment modality for NAFLD.

\section{Abbreviation list}

ACE, abundance-based coverage estimator; ALP, alkaline phosphatase; ALT, alanine aminotransferase; ANOVA, analysis of variance; AST, aspartate aminotransferase; BSEP, bile salt export pump; CA, cholic acid; CDCA, chenodeoxycholic acid; Ct, cycle threshold; CYP7A1, cholesterol $7 \alpha-$ hydroxylase; CYP8B1, sterol 12 $\alpha$-hydroxylase; DCA, deoxycholic acid; Fgf15, fibroblast growth factor 15; FMT, fecal microbiota transplantation; FXR, farnesoid $\mathrm{X}$ receptor; GAPDH, glyceraldehyde phosphate dehydrogenase; GGT, $\gamma$ glutamyl transpeptidase; HE, hematoxylin-eosin; HFD, high fat diet; HLD-C, high-density lipoprotein-cholesterol; IL, interleukin; LBP, lipopolysaccharide-binding protein; LCA, lithocholic acid; LDA, linear discriminant analysis; LDL-C, low-density lipoprotein-cholesterol; LEfSe, linear discriminant analysis with effect size; LPS, lipopolysaccharide; MCA, muricholic acid; MRM, multiple reaction monitor; MS, mass spectrometry; NAFLD, non-alcoholic fatty liver disease; NASH, non-alcoholic steatohepatitis; NIH, National Institutes of Health; NMDS, nonmetric multidimensional scaling; $\mathrm{PBC}$, primary biliary cholangitis; $\mathrm{PC} 1$, the first principal component; PCoA, principal coordinate analysis; RT-qPCR, reverse transcription quantitative real-time PCR; SCFA, short chain fatty acid; SD, standard deviation; SHP, small heterodimer partner; TB, total bilirubin; TBS, Tris-buffered saline; TCA, tauro-cholic acid; TCDCA, tauro-chenodeoxycholic;
TNF- $\alpha$, tumor necrosis factor- $\alpha$; T $\alpha M C A$, tauro- $\alpha-$ muricholic acid; T $\beta$ MCA, tauro- $\beta$-muricholic acid; UDCA, ursodeoxycholic acid; UPLC, ultra-high-performance liquid chromatography; ZO-1, tight junction protein-1; $\alpha \mathrm{MCA}, \alpha-$ muricholic acid; $\beta \mathrm{MCA}, \beta$-muricholic acid.

\section{Acknowledgments}

This study was supported by the National Natural Science Foundation of China (No. 81000968, No. 81101540, No. 81101637, No. 81172273, No. 81272388, No. 81301820, No. 81472673), the Fund of Shanghai Science and Technology Commission (16ZR1406100,15410710100), the Doctoral Fund of Ministry of Education of China (20120071110058), and the National Clinical Key Special Subject of China. The authors would like to thank the members of Professor XiZhong Shen's laboratory for helpful discussions and critical reading of the manuscript.

\section{Disclosure}

The authors report no conflicts of interest in this work.

\section{References}

1. Rinella ME. Nonalcoholic fatty liver disease: a systematic review. JAMA. 2015;313:2263-2273. doi:10.1001/jama.2015.5370

2. Wruck W, Graffmann N, Kawala MA, Adjaye J. Concise review: current status and future directions on research related to nonalcoholic fatty liver disease. Stem Cells. 2017;35:89-96. doi:10.1002/stem.2454

3. Cohen JC, Horton JD, Hobbs HH. Human fatty liver disease: old questions and new insights. Science. 2011;332:1519-1523. doi:10.1126/science. 1204265

4. Chalasani N, Younossi Z, Lavine JE, et al. The diagnosis and management of non-alcoholic fatty liver disease: practice guideline by the American Gastroenterological Association, American Association for the Study of Liver Diseases, and American College of Gastroenterology. Gastroenterology. 2012;142:1592-1609. doi:10.10 53/j.gastro.2012.04.001

5. Han CY. Update on FXR biology: promising therapeutic target? Int J Mol Sci. 2018;19:2069. doi:10.3390/ijms19072069

6. Chavez-Talavera O, Tailleux A, Lefebvre P, Staels B. Bile acid control of metabolism and inflammation in obesity, type 2 diabetes, dyslipidemia, and nonalcoholic fatty liver disease. Gastroenterology. 2017;152:1679-1694. doi:10.1053/j.gastro.2017.01.055

7. Morrison MC, Verschuren L, Salic K, et al. Obeticholic acid modulates serum metabolites and gene signatures characteristic of human NASH and attenuates inflammation and fibrosis progression in ldlr-/-.leiden mice. Hepatol Commun. 2018;2:1513-1532. doi:10.1002/hep4.1270

8. Kakiyama G, Pandak WM, Gillevet PM, et al. Modulation of the fecal bile acid profile by gut microbiota in cirrhosis. $J$ Hepatol. 2013;58:949-955. doi:10.1016/j.jhep.2013.01.003

9. Hooper LV, Gordon JI. Commensal host-bacterial relationships in the gut. Science. 2001;292:1115-1118.

10. Kau AL, Ahern PP, Griffin NW, Goodman AL, Gordon JI. Human nutrition, the gut microbiome and the immune system. Nature. 2011;474:327-336. doi:10.1038/nature10213

11. Tremaroli V, Backhed F. Functional interactions between the gut microbiota and host metabolism. Nature. 2012;489:242-249. doi:10.1038/nature 11552 
12. Bakhtiar SM, LeBlanc JG, Salvucci E, et al. Implications of the human microbiome in inflammatory bowel diseases. FEMS Microbiol Lett. 2013;342:10-17. doi:10.1111/1574-6968.12111

13. Vijay-Kumar M, Aitken JD, Carvalho FA, et al. Metabolic syndrome and altered gut microbiota in mice lacking Toll-like receptor 5. Science. 2010;328:228-231. doi:10.1126/science.1179721

14. Nicholson JK, Holmes E, Kinross J, et al. Host-gut microbiota metabolic interactions. Science. 2012;336:1262-1267. doi:10.1126/science.1223813

15. Perry RJ, Peng L, Barry NA, et al. Acetate mediates a microbiome-brain-beta-cell axis to promote metabolic syndrome. Nature. 2016;534:213-217. doi:10.1038/nature18309

16. Liu HN, Wu H, Chen YZ, Chen Y-J, Shen X-Z, Liu -T-T. Altered molecular signature of intestinal microbiota in irritable bowel syndrome patients compared with healthy controls: a systematic review and meta-analysis. Dig Liver Dis. 2017;49:331-337. doi:10.1016/j. dld.2017.01.142

17. Chen YJ, Wu H, Wu SD, et al. Parasutterella, in association with irritable bowel syndrome and intestinal chronic inflammation. $J$ Gastroenterol Hepatol. 2018;33:1844-1852. doi:10.1111/jgh.14281

18. Xie G, Wang Y, Wang X, et al. Profiling of serum bile acids in a healthy Chinese population using UPLC-MS/MS. J Proteome Res. 2015;14:850-859. doi:10.1021/pr500920q

19. Byrne CD, Targher G. NAFLD: a multisystem disease. J Hepatol. 2015;62:S47-S64.

20. Tilg H, Moschen AR. Evolution of inflammation in nonalcoholic fatty liver disease: the multiple parallel hits hypothesis. Hepatology. 2010;52:1836-1846. doi:10.1002/hep.24001

21. Zafrani ES. Non-alcoholic fatty liver disease: an emerging pathological spectrum. Virchows Arch. 2004;444:3-12. doi:10.1007/s00428003-0943-7

22. Fuchs M. Non-alcoholic Fatty liver disease: the bile Acid-activated farnesoid $\mathrm{x}$ receptor as an emerging treatment target. J Lipids. 2012;2012:934396. doi:10.1155/2012/934396

23. Manley S, Ding W. Role of farnesoid X receptor and bile acids in alcoholic liver disease. Acta Pharm Sin B. 2015;5:158-167. doi:10.1016/j.apsb.2014.12.011

24. Neuschwander-Tetri BA, Loomba R, Sanyal AJ, et al. Farnesoid $\mathrm{X}$ nuclear receptor ligand obeticholic acid for non-cirrhotic, non-alcoholic steatohepatitis (FLINT): a multicentre, randomised, placebo-controlled trial. Lancet. 2015;385:956-965. doi:10.1016/ S0140-6736(14)61933-4

25. Cipriani S, Mencarelli A, Palladino G, Fiorucci S. FXR activation reverses insulin resistance and lipid abnormalities and protects against liver steatosis in Zucker $(\mathrm{fa} / \mathrm{fa})$ obese rats. J Lipid Res. 2010;51:771-784. doi:10.1194/jlr.M001602

26. Fickert P, Fuchsbichler A, Moustafa T, et al. Farnesoid X receptor critically determines the fibrotic response in mice but is expressed to a low extent in human hepatic stellate cells and periductal myofibroblasts. Am J Pathol. 2009;175:2392-2405. doi:10.2353/ ajpath.2009.090114

27. Verbeke L, Farre R, Trebicka J, et al. Obeticholic acid, a farnesoid $\mathrm{X}$ receptor agonist, improves portal hypertension by two distinct pathways in cirrhotic rats. Hepatology. 2014;59:2286-2298. doi:10.1002/hep.26939
28. Armstrong MJ, Newsome PN. Trials of obeticholic acid for non-alcoholic steatohepatitis. Lancet. 2015;386:28.

29. Mao Y, Zhou C, Zhu L, et al. Identification and expression analysis on bactericidal permeability-increasing protein (BPI)/lipopolysaccharide-binding protein (LBP) of ark shell, Scapharca broughtonii. Fish Shellfish Immunol. 2013;35:642-652. doi:10.1016/j.fsi.2013.05.025

30. Cani PD, Bibiloni R, Knauf $\mathrm{C}$, et al. Changes in gut microbiota control metabolic endotoxemia-induced inflammation in high-fat diet-induced obesity and diabetes in mice. Diabetes. 2008;57:1470-1481. doi:10.2337/db07-1403

31. Pendyala S, Walker JM, Holt PR. A high-fat diet is associated with endotoxemia that originates from the gut. Gastroenterology. 2012;142:1100-1101. doi:10.1053/j.gastro.2012.01.034

32. Ruiz AG, Casafont F, Crespo J, et al. Lipopolysaccharide-binding protein plasma levels and liver TNF-alpha gene expression in obese patients: evidence for the potential role of endotoxin in the pathogenesis of non-alcoholic steatohepatitis. Obes Surg. 2007;17:1374-1380. doi:10.1007/s11695-007-9243-7

33. Sakura T, Morioka T, Shioi A, et al. Lipopolysaccharide-binding protein is associated with arterial stiffness in patients with type 2 diabetes: a cross-sectional study. Cardiovasc Diabetol. 2017;16:62. doi:10.1186/s12933-017-0624-5

34. Luo Y, Liu M. Adiponectin: a versatile player of innate immunity. J Mol Cell Biol. 2016;8:120-128. doi:10.1093/jmcb/mjw012

35. Sayin SI, Wahlstrom A, Felin J, et al. Gut microbiota regulates bile acid metabolism by reducing the levels of tauro-beta-muricholic acid, a naturally occurring FXR antagonist. Cell Metab. 2013;17:225-235. doi:10.1016/j.cmet.2013.01.003

36. Jenq RR, Taur Y, Devlin SM, et al. Intestinal Blautia is associated with reduced death from graft-versus-host disease. Biol Blood Marrow Transplant. 2015;21:1373-1383. doi:10.1016/j. bbmt.2015.04.016

37. Byrne CS, Chambers ES, Morrison DJ, Frost G. The role of short chain fatty acids in appetite regulation and energy homeostasis. Int J Obes (Lond). 2015;39:1331-1338. doi:10.1038/ijo.2015.84

38. Rios-Covian D, Ruas-Madiedo P, Margolles A, et al. Intestinal short chain fatty acids and their link with diet and human health. Front Microbiol. 2016;7:185. doi:10.3389/fmicb.2016.00185

39. Hooper LV, Littman DR, Macpherson AJ. Interactions between the microbiota and the immune system. Science. 2012;336:1268-1273. doi: $10.1126 /$ science. 1223490

40. Maynard CL, Elson CO, Hatton RD, Weaver CT. Reciprocal interactions of the intestinal microbiota and immune system. Nature. 2012;489:231-241. doi:10.1038/nature11551

41. Koh A, De Vadder F, Kovatcheva-Datchary P, Bäckhed F. From dietary fiber to host physiology: short-chain fatty acids as key bacterial metabolites. Cell. 2016;165:1332-1345. doi:10.1016/j.cell.2016.05.041

42. Dupont J, Oh SY, O'Deen LA, Geller S. Cholanoic (bile) acids in hepatic and nonhepatic tissues of miniature swine. Lipids. 1974;9:294-297.

43. Inagaki T, Choi M, Moschetta A, et al. Fibroblast growth factor 15 functions as an enterohepatic signal to regulate bile acid homeostasis. Cell Metab. 2005;2:217-225. doi:10.1016/j.cmet.2005.09.001 


\section{Supplementary materials}

Table SI cDNA target genes and primers used for quantitative real-time PCR

\begin{tabular}{|c|c|c|}
\hline Gene & Forward primer $\left(5^{\prime}-3^{\prime}\right)$ & Reverse primer $\left(3^{\prime}-5^{\prime}\right)$ \\
\hline$\beta$-actin & GGCTGTATTCCCCTCCATCG & CCAGTTGGTAACAATGCCATGT \\
\hline ZO-I & GCCGCTAAGAGCACAGCAA & TCCССACTCTGAAAATGAGGA \\
\hline Occludin & TTGAAAGTCCACCTCCTTACAGA & CCGGATAAAAAGAGTACGCTGG \\
\hline TNF- $\alpha$ & СССТСАСАСТСАGАТСАТСТTСТ & GCTACGACGTGGGCTACAG \\
\hline IL-I $\beta$ & GAAATGCCACCTTTTGACAGTG & TGGATGCTCTCATCAGGACAG \\
\hline IL-10 & GCTCTTACTGACTGGCATGAG & CGCAGCTCTAGGAGCATGTG \\
\hline SHP & TGGGTCCCAAGGAGTATGC & GCTCCAAGACTTCACACAGTG \\
\hline CYP7AI & GGGATTGCTGTGGTAGTGAGC & GGTATGGAATCAACCCGTTGTC \\
\hline CYP8BI & ССTCTGGACAAGGGTTTTGTG & GCACCGTGAAGACATCCCC \\
\hline BSEP & TCTGACTCAGTGATTCTTCGCA & CCCATAAACATCAGCCAGTTGT \\
\hline Fgfl5 & ATGGCGAGAAAGTGGAACGG & CTGACACAGACTGGGATTGCT \\
\hline
\end{tabular}


A

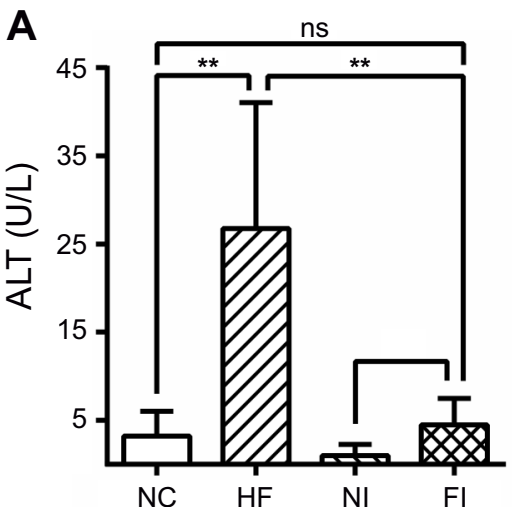

D

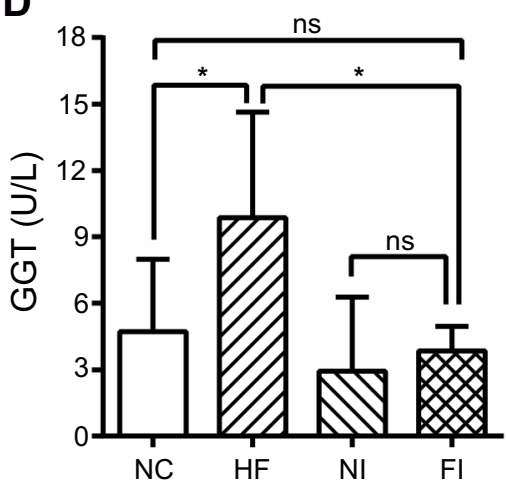

G

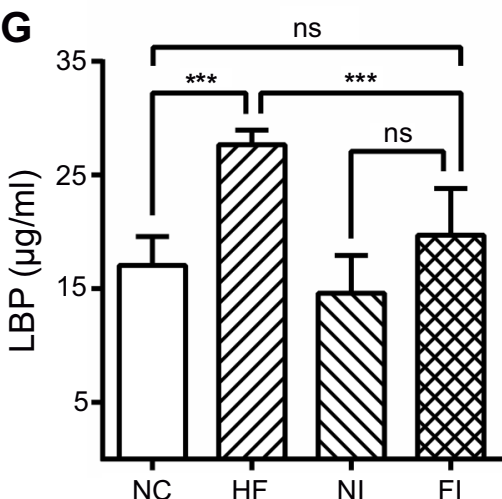

B

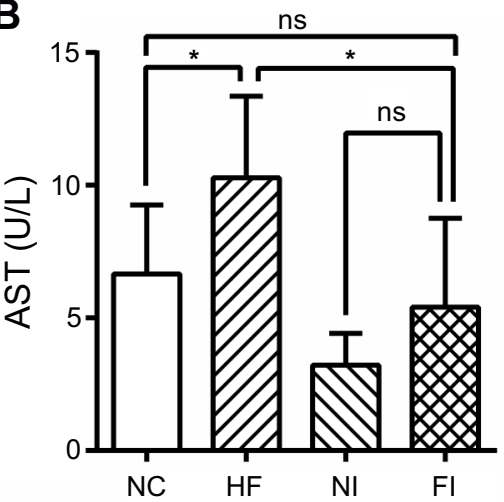

$\mathbf{E}$

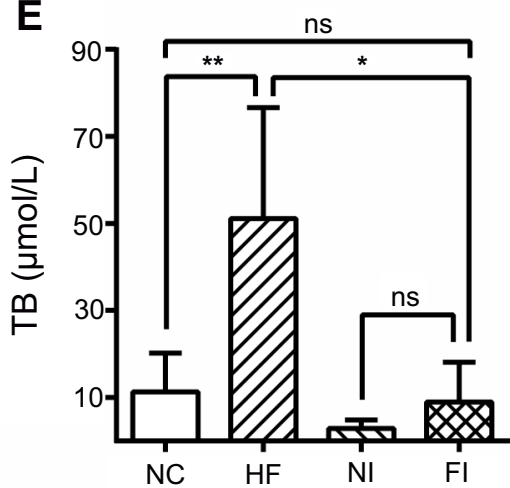

H

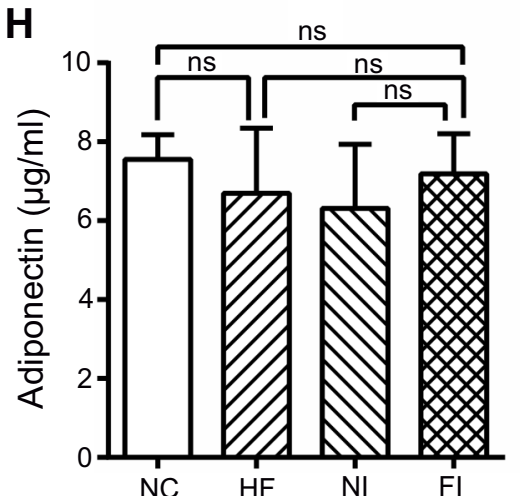

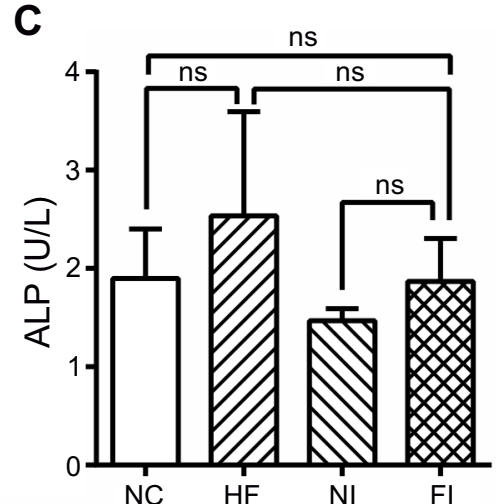

$\mathbf{F}$
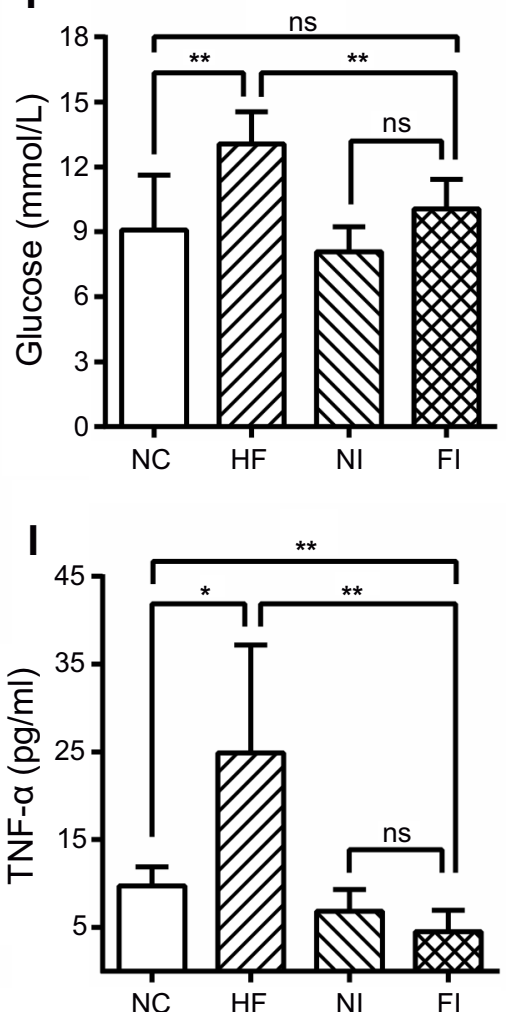

Figure SI Serum liver function, glucose and inflammatory mediators. (A) Serum ALT, (B) AST, (C) ALP, (D) GGT, (E) TB, (F) glucose, (G) LBP, (H) adiponectin and (I) TNF- $\alpha . * P<0.05 ; * * P<0.01 ; * * *<<0.001$; ns, not statistically significant.

Abbreviations: ALT, alanine aminotransferase; AST, aspartate aminotransferase; ALP, alkaline phosphatase; GGT, gamma-glutamyl transpeptidase; TB, total bilirubin; LBP, lipopolysaccharide-binding protein; TNF- $\alpha$, tumor necrosis factor-alpha. 
Table S2 The differential expressed bacteria among three groups from phylum to genus level

\begin{tabular}{|c|c|c|c|c|c|}
\hline Classification & Name & NC group & HF group & FI group & $P$-value \\
\hline Order & Gastranaerophilales & $0.405 \pm 0.489$ & $0.000 \pm 0.000$ & $0.000 \pm 0.000$ & $<0.001$ \\
\hline Genus & Anaeroplasma & $0.052 \pm 0.060$ & $0.000 \pm 0.000$ & $0.000 \pm 0.000$ & $<0.001$ \\
\hline Genus & Akkermansia & $4.638 \pm 7.420$ & $0.054 \pm 0.091$ & $0.000 \pm 0.000$ & $<0.001$ \\
\hline Genus & Roseburia & $0.075 \pm 0.085$ & $0.258 \pm 0.380$ & $1.336 \pm 1.006$ & 0.002 \\
\hline Genus & Ruminococcaceae_UCG-0I4 & $0.396 \pm 0.303$ & $0.00 I \pm 0.003$ & $0.009 \pm 0.020$ & 0.003 \\
\hline Genus & Aeromonas & $0.012 \pm 0.020$ & $0.040 \pm 0.039$ & $0.000 \pm 0.000$ & 0.004 \\
\hline Genus & Bacteroidales_S24-7_group & $40.660 \pm 9.725$ & $15.230 \pm 12.700$ & $13.920 \pm 12.500$ & 0.005 \\
\hline Family & Family_XIII & $0.010 \pm 0.009$ & $0.000 \pm 0.000$ & $0.001 \pm 0.003$ & 0.005 \\
\hline Class & Cyanobacteria & $0.002 \pm 0.006$ & $0.067 \pm 0.147$ & $0.000 \pm 0.000$ & 0.006 \\
\hline Genus & Mucispirillum & $0.499 \pm 0.789$ & $0.170 \pm 0.271$ & $2.793 \pm 2.644$ & 0.007 \\
\hline Order & Mollicutes_RF9 & $0.154 \pm 0.132$ & $0.000 \pm 0.000$ & $0.006 \pm 0.010$ & 0.007 \\
\hline Genus & Weissella & $0.007 \pm 0.012$ & $0.014 \pm 0.011$ & $0.001 \pm 0.001$ & 0.007 \\
\hline Genus & Turicibacter & $0.288 \pm 0.554$ & $0.000 \pm 0.000$ & $1.216 \pm 2.075$ & 0.010 \\
\hline Genus & Allobaculum & $0.949 \pm 0.949$ & $0.000 \pm 0.000$ & $0.198 \pm 0.519$ & 0.013 \\
\hline Family & Sphingomonadaceae & $0.001 \pm 0.002$ & $0.012 \pm 0.013$ & $0.001 \pm 0.002$ & 0.017 \\
\hline Family & Rhodospirillaceae & $0.534 \pm 0.836$ & $0.452 \pm 0.834$ & $0.013 \pm 0.028$ & 0.017 \\
\hline Genus & Ureaplasma & $0.000 \pm 0.000$ & $0.052 \pm 0.072$ & $0.000 \pm 0.000$ & 0.018 \\
\hline Genus & Tyzzerella_3 & $0.000 \pm 0.000$ & $0.011 \pm 0.023$ & $0.000 \pm 0.000$ & 0.018 \\
\hline Family & Bacillaceae & $0.000 \pm 0.000$ & $0.006 \pm 0.010$ & $0.000 \pm 0.000$ & 0.018 \\
\hline Genus & Sporosarcina & $0.000 \pm 0.000$ & $0.006 \pm 0.010$ & $0.000 \pm 0.000$ & 0.018 \\
\hline Genus & Lachnoclostridium & $0.378 \pm 0.285$ & $0.229 \pm 0.131$ & $1.494 \pm 1.548$ & 0.019 \\
\hline Genus & Ruminiclostridium & $3.337 \pm 2.693$ & $0.85 I \pm 1.189$ & $4.913 \pm 3.294$ & 0.021 \\
\hline Genus & [Ruminococcus]_torques_group & $0.000 \pm 0.000$ & $0.001 \pm 0.002$ & $0.045 \pm 0.094$ & 0.021 \\
\hline Genus & Dialister & $0.000 \pm 0.000$ & $0.008 \pm 0.009$ & $0.001 \pm 0.002$ & 0.021 \\
\hline Genus & Acetatifactor & $0.091 \pm 0.108$ & $0.00 I \pm 0.003$ & $0.058 \pm 0.130$ & 0.023 \\
\hline Genus & [Eubacterium]_brachy_group & $0.004 \pm 0.006$ & $0.003 \pm 0.003$ & $0.043 \pm 0.068$ & 0.023 \\
\hline Family & Lachnospiraceae & $1.383 \pm 1.092$ & $2.028 \pm 2.269$ & $4.238 \pm 2.268$ & 0.024 \\
\hline Genus & Ruminococcaceae_NK4A214_group & $0.056 \pm 0.069$ & $0.001 \pm 0.003$ & $0.066 \pm 0.113$ & 0.025 \\
\hline Genus & Blautia & $1.349 \pm 1.282$ & $3.492 \pm 8.683$ & $5.248 \pm 3.508$ & 0.025 \\
\hline Genus & Coprococcus_I & $0.26 I \pm 0.325$ & $0.25 I \pm 0.640$ & $0.365 \pm 0.135$ & 0.027 \\
\hline Genus & Eisenbergiella & $0.027 \pm 0.059$ & $0.000 \pm 0.000$ & $0.000 \pm 0.000$ & 0.029 \\
\hline Family & Coriobacteriaceae & $0.015 \pm 0.026$ & $0.000 \pm 0.000$ & $0.000 \pm 0.000$ & 0.029 \\
\hline Family & Erysipelotrichaceae & $0.007 \pm 0.014$ & $0.000 \pm 0.000$ & $0.000 \pm 0.000$ & 0.029 \\
\hline Family & HSB_OF53-F07 & $0.005 \pm 0.006$ & $0.000 \pm 0.000$ & $0.000 \pm 0.000$ & 0.029 \\
\hline Genus & Anaerofilum & $0.004 \pm 0.009$ & $0.000 \pm 0.000$ & $0.000 \pm 0.000$ & 0.029 \\
\hline Genus & Raoultella & $0.004 \pm 0.009$ & $0.017 \pm 0.032$ & $0.000 \pm 0.000$ & 0.030 \\
\hline Genus & Lachnospiraceae_UCG-006 & $0.078 \pm 0.077$ & $0.029 \pm 0.060$ & $0.337 \pm 0.447$ & 0.033 \\
\hline Genus & Lachnospiraceae_FCS020_group & $0.030 \pm 0.036$ & $0.010 \pm 0.023$ & $0.044 \pm 0.037$ & 0.033 \\
\hline Genus & [Eubacterium]_xylanophilum_group & $0.001 \pm 0.002$ & $0.000 \pm 0.000$ & $0.07 I \pm 0.087$ & 0.033 \\
\hline Genus & Anaerotruncus & $0.892 \pm 0.527$ & $1.259 \pm 1.627$ & $3.436 \pm 1.881$ & 0.033 \\
\hline Genus & Christensenella & $0.015 \pm 0.016$ & $0.000 \pm 0.001$ & $0.004 \pm 0.007$ & 0.033 \\
\hline Family & Christensenellaceae & $0.011 \pm 0.013$ & $0.027 \pm 0.032$ & $0.048 \pm 0.038$ & 0.037 \\
\hline Genus & Pseudomonas & $0.005 \pm 0.009$ & $0.033 \pm 0.072$ & $0.002 \pm 0.004$ & 0.039 \\
\hline Family & Coriobacteriaceae & $0.016 \pm 0.014$ & $0.002 \pm 0.003$ & $0.003 \pm 0.007$ & 0.041 \\
\hline Genus & Ruminococcaceae_UCG-0I0 & $0.187 \pm 0.209$ & $0.015 \pm 0.021$ & $0.017 \pm 0.028$ & 0.042 \\
\hline Genus & Alloprevotella & $3.593 \pm 4.012$ & $12.570 \pm 9.983$ & $2.045 \pm 4.448$ & 0.043 \\
\hline Genus & Peptoclostridium & $0.128 \pm 0.274$ & $0.736 \pm 1.402$ & $0.000 \pm 0.000$ & 0.045 \\
\hline Genus & Pediococcus & $0.003 \pm 0.004$ & $0.009 \pm 0.014$ & $0.000 \pm 0.000$ & 0.045 \\
\hline Family & Lachnospiraceae & $0.800 \pm 0.512$ & $0.691 \pm 0.987$ & $2.591 \pm 1.672$ & 0.045 \\
\hline Genus & Phascolarctobacterium & $0.003 \pm 0.007$ & $0.007 \pm 0.008$ & $0.000 \pm 0.000$ & 0.046 \\
\hline Genus & Ruminococcaceae_UCG-0I3 & $0.05 I \pm 0.08 I$ & $0.002 \pm 0.005$ & $0.001 \pm 0.001$ & 0.047 \\
\hline
\end{tabular}

Notes: Only taxa with a linear discriminant analysis (LDA) significant threshold $>3$ and $P$-value $<0.05$ are shown. 

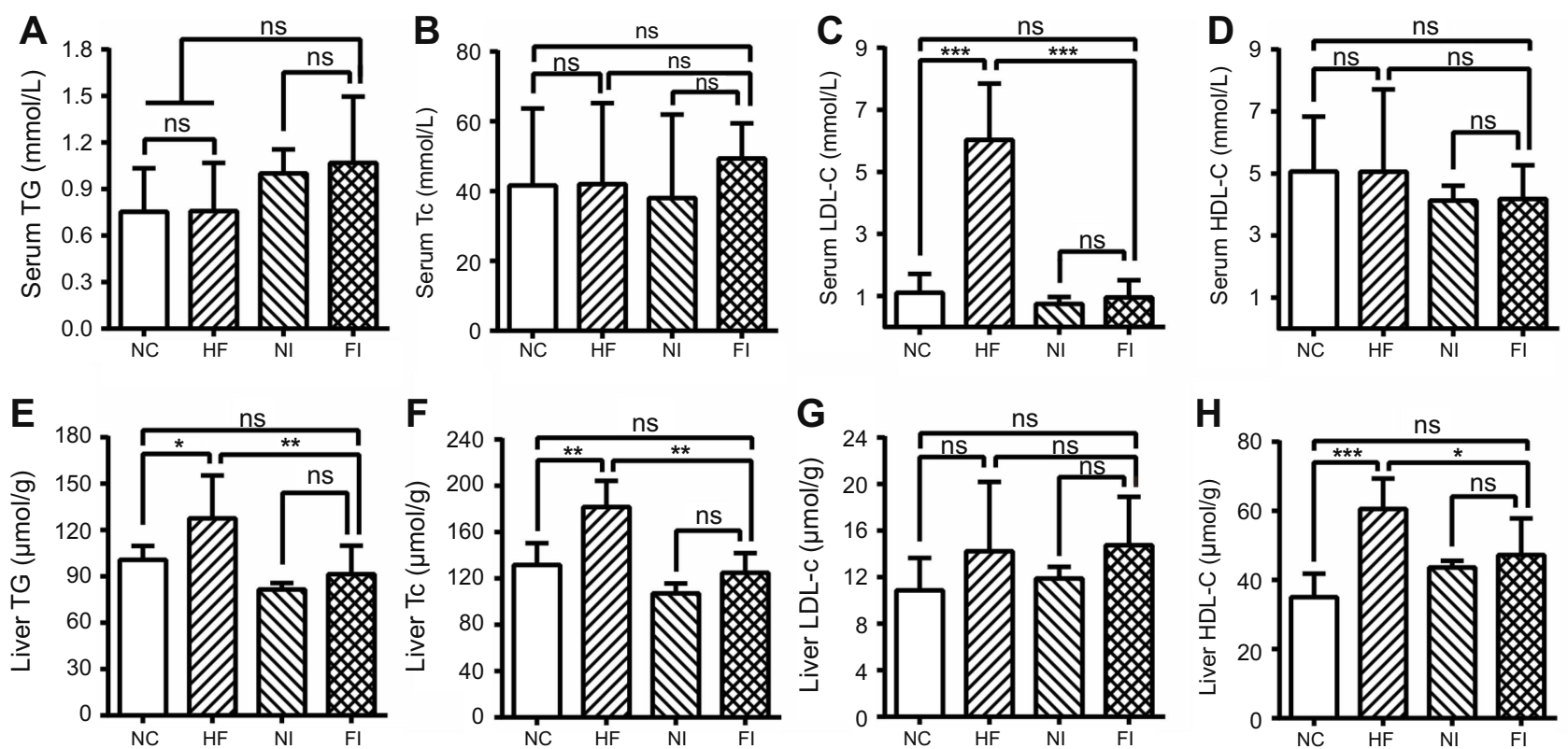

Figure S2 Serum and liver lipids. (A) Serum triglyceride, (B) total cholesterol, (C) LDL-C and (D) HDL-C. (E) Liver triglycerides, (F) cholesterol, (G) LDL-C and (H) HDL-C. $* P<0.05 ; * * P<0.01$; $* * * P<0.001$; ns, not statistically significant.

Abbreviations: TG, triglyceride; TC, total cholesterol; LDL-C, low-density lipoprotein-cholesterol; HDL-C, high-density lipoprotein-cholesterol. 
Table S3 Bile acid levels in liver, ileum, cecum, feces and gallbladder

\begin{tabular}{|c|c|c|c|c|}
\hline Position & Bile acid & NC group & HF group & FI group \\
\hline Liver & $\begin{array}{l}\text { CA } \\
\text { CDCA } \\
\text { DCA } \\
\text { LCA } \\
\text { MCA } \\
\text { UDCA } \\
\alpha M C A \\
\beta M C A \\
\text { TCA } \\
\text { TCDCA } \\
\text { TLCA } \\
\text { TUDCA } \\
\text { TaMCA } \\
\text { T } \beta M C A \\
\text { Total BAs }\end{array}$ & $\begin{array}{l}1.30 \pm 1.10 \\
21.53 \pm 10.70 \\
0.14 \pm 0.11 \\
\text { nd } \\
0.27 \pm 0.11 \\
0.93 \pm 0.69 \\
22.95 \pm 17.37 \\
7.94 \pm 5.55 \\
68.51 \pm 76.84 \\
3.18 \pm 2.76 \\
0.12 \pm 0.11 \\
4.86 \pm 6.09 \\
32.10 \pm 46.82 \\
14.80 \pm 23.85 \\
197.89 \pm 143.09\end{array}$ & $\begin{array}{l}0.63 \pm 0.55 \\
18.20 \pm 13.85 \\
0.07 \pm 0.02 \\
\text { nd } \\
0.10 \pm 0.09 * \\
0.27 \pm 0.25 \\
22.89 \pm 16.84 \\
3.60 \pm 2.32 \\
115.17 \pm 137.28 \\
6.49 \pm 3.62 \\
0.11 \pm 0.05 \\
3.03 \pm 3.36 \\
58.80 \pm 86.80 \\
8.98 \pm 11.31 \\
252.89 \pm 244.44\end{array}$ & $\begin{array}{l}\text { nd } \\
22.05 \pm 12.14 \\
\text { nd } \\
\text { nd } \\
0.05 \pm 0.03 \\
0.53 \pm 0.28 \\
27.73 \pm 14.94 \\
3.25 \pm 1.21 \\
1.76 \pm 1.66 \\
2.79 \pm 1.12 \\
0.03 \pm 0.04 \# \\
1.1 \pm 0.75 \\
10.24 \pm 5.37 \\
3.35 \pm 1.59 \\
89.21 \pm 38.65\end{array}$ \\
\hline Ileum & $\begin{array}{l}\text { CA } \\
\text { CDCA } \\
\text { DCA } \\
\text { LCA } \\
\text { MCA } \\
\text { UDCA } \\
\alpha M C A \\
\beta M C A \\
\text { TCA } \\
\text { TCDCA } \\
\text { TLCA } \\
\text { TUDCA } \\
\text { TaMCA } \\
\text { T } \beta M C A \\
\text { Total BAs }\end{array}$ & $\begin{array}{l}3655.42 \pm 5968.83 \\
52.64 \pm 46.76 \\
86.57 \pm|3| .48 \\
\mid 8.95 \pm 3.80 \\
219.57 \pm 37.63 \\
|28.33 \pm| 46.25 \\
316.44 \pm 495.48 \\
|39| .10 \pm \mid 790.87 \\
20,867.12 \pm 39,538.84 \\
\mid 584.02 \pm 3398.33 \\
10.75 \pm 9.05 \\
1030.22 \pm 2024.30 \\
4 \mid, 864.44 \pm 67,439.39 \\
7 \mid 56.25 \pm 9875.58 \\
80,889.06 \pm \mid 26,204.67\end{array}$ & $\begin{array}{l}5727.67 \pm 579 \mid .30 \\
81.20 \pm 57.75 \\
108.08 \pm \mid 16.00 \\
21.25 \pm 2.45 \\
225.58 \pm \mid 3.33 \\
91.72 \pm 53.22 \\
268.56 \pm 29 \mid .12 \\
|352.82 \pm| 46 \mid .88 \\
|8,877.3| \pm 21,000.98 \\
809.76 \pm|00| . \mid 4 \\
3.99 \pm 5.16 \\
581.06 \pm 578.49 \\
24,195.60 \pm 22,949.56 \\
4996.74 \pm 4682.02 \\
58,426.30 \pm 51,657.59\end{array}$ & $\begin{array}{l}235.92 \pm 356.7 \\
57.36 \pm 33.83 \\
24.37 \pm 6.6 \\
17.22 \pm 1.82 \# \\
198.56 \pm 15.19 \# \\
163.84 \pm 103.19 \\
224.33 \pm 235.75 \\
679.22 \pm 804.18 \\
12,690.96 \pm 84 \mid 5.15 \\
5459.8 \pm 4454.6 \# \\
7.38 \pm 9.21 \\
3371.65 \pm 2777.77 \# \\
99,021.06 \pm 67,192.1 \# \\
16,855.76 \pm \mid 1,888.44 \# \\
\mid 42,015.24 \pm 88,462.65\end{array}$ \\
\hline Cecum & $\begin{array}{l}\text { CA } \\
\text { CDCA } \\
\text { DCA } \\
\text { LCA } \\
\text { MCA } \\
\text { UDCA } \\
\alpha M C A \\
\beta M C A \\
\text { TCA } \\
\text { TCDCA } \\
\text { TLCA } \\
\text { TUDCA } \\
\text { TaMCA } \\
\text { T } \beta M C A \\
\text { Total BAs }\end{array}$ & $\begin{array}{l}363.61 \pm 751.76 \\
\text { nd } \\
696.65 \pm 420.40 \\
51.98 \pm 30.72 \\
29.66 \pm 7.44 \\
385.79 \pm 191.63 \\
411.94 \pm 390.7 \mid \\
1167.99 \pm 998.12 \\
16.90 \pm|7.4| \\
1.34 \pm 1.44 \\
0.18 \pm 0.18 \\
1.62 \pm 1.25 \\
52.88 \pm 51.51 \\
\text { nd } \\
4227.03 \pm 1789.49\end{array}$ & $\begin{array}{l}33.12 \pm 24.42 \\
\text { nd } \\
10 \mid 3.59 \pm 663.00 \\
89.22 \pm 57.90 \\
25.60 \pm 7.75 \\
137.01 \pm 57.70^{*} \\
|87.22 \pm| 33.04 \\
437.52 \pm 3 \mid 2.95 \\
3.14 \pm 0.87 \\
0.29 \pm 0.17 \\
0.48 \pm 0.4 \mid \\
0.72 \pm 0.40 \\
20.86 \pm \mid 3.78 \\
\text { nd } \\
2496.44 \pm|44| .66\end{array}$ & $\begin{array}{l}1.95 \pm 1.50 \# \\
132.42 \pm 7.93 \\
10.54 \pm 7.69 \# \\
19.08 \pm 19.30 \\
21.52 \pm 1.80 \\
26.37 \pm \mid 2.00 \# \\
47.71 \pm 33.72 \\
142.58 \pm 145.27 \\
\text { nd } \\
2.12 \pm 2.88 \\
0.34 \pm 0.16 \\
1.32 \pm 1.21 \\
35.48 \pm 15.59 \\
\text { nd } \\
677.24 \pm 249.4 \mid \#\end{array}$ \\
\hline
\end{tabular}

(Continued) 
Table S3 (Continued).

\begin{tabular}{|c|c|c|c|c|}
\hline Position & Bile acid & NC group & HF group & FI group \\
\hline Feces & $\begin{array}{l}\text { CA } \\
\text { CDCA } \\
\text { DCA } \\
\text { LCA } \\
\text { MCA } \\
\text { UDCA } \\
\alpha M C A \\
\beta M C A \\
\text { TCA } \\
\text { TCDCA } \\
\text { TLCA } \\
\text { TUDCA } \\
\text { T } \alpha \text { MCA } \\
\text { T } \beta M C A \\
\text { Total BAs }\end{array}$ & $\begin{array}{l}\mid 85.62 \pm 364.44 \\
|0.05 \pm| 3.57 \\
936.16 \pm 1074.67 \\
80.14 \pm 37.92 \\
25.37 \pm 34.42 \\
242.49 \pm 354.99 \\
205.80 \pm 295.84 \\
594.02 \pm 7 \mid 6.96 \\
\mid 4.43 \pm 9.90 \\
0.44 \pm 0.26 \\
0.03 \pm 0.03 \\
8.60 \pm 1.02 \\
8.56 \pm 6.94 \\
\text { nd } \\
3488.8 \mid \pm 3843.34\end{array}$ & $\begin{array}{l}20.29 \pm 10.75 \\
2.15 \pm 1.10 \\
620.78 \pm 521.31 \\
51.43 \pm 47.79 \\
6.68 \pm 2.32 \\
29.15 \pm 20.92 \\
48.18 \pm 52.85 \\
160.50 \pm 183.33 \\
18,954.99 \pm 30,581.17 \\
\text { nd } \\
0.39 \pm 0.43 \\
8.02 \pm 0.47 \\
8.40 \pm 4.93 \\
\text { nd } \\
20,455.36 \pm 29,744.41\end{array}$ & $\begin{array}{l}225.34 \pm 260.38 \\
51.03 \pm 34.29 \# \\
36 \mid \pm 302.73 \\
93.47 \pm 67.19 \\
|7.11 \pm| 5.77 \\
89.68 \pm 40.49 \# \\
355.82 \pm \mid 84.09 \# \\
656.64 \pm 248.46 \# \\
|1.2| \pm 7.18 \\
\text { nd } \\
0.34 \pm 0.34 \\
9.37 \pm 0.42 \# \\
|4.94 \pm| 3.58 \\
\text { nd } \\
2994.7| \pm| 480.3 \mid\end{array}$ \\
\hline Gallbladder & $\begin{array}{l}\text { CA } \\
\text { CDCA } \\
\text { DCA } \\
\text { LCA } \\
\text { MCA } \\
\text { UDCA } \\
\alpha M C A \\
\beta M C A \\
\text { TCA } \\
\text { TCDCA } \\
\text { TLCA } \\
\text { TUDCA } \\
\text { TaMCA } \\
\text { T } \beta M C A \\
\text { Total BAs }\end{array}$ & $\begin{array}{l}4555.48 \pm 6389.83 \\
25,97|.27 \pm 2|, 299.54 \\
70.59 \pm 47.93 \\
\text { nd } \\
\text { nd } \\
\text { nd } \\
|5,28| .35 \pm \mid 2,652.73 \\
3|, 324 .| 3 \pm 25,352.78 \\
252,863.2| \pm 346,530.1| \\
3 \mid 85.57 \pm 4637.84 \\
|29.07 \pm| 24.74 \\
3896.3| \pm 64| 8.3 \mid \\
232,323.70 \pm 3|2,54| .70 \\
\text { nd } \\
577,4 \mid 3.39 \pm 734,299.67\end{array}$ & $\begin{array}{l}245.18 \pm 145.93 \\
38,097.67 \pm 19,471.68 \\
65.23 \pm 40.24 \\
\text { nd } \\
\text { nd } \\
\text { nd } \\
21,312.99 \pm 10,906.48 \\
43,529.57 \pm 22,317.74 \\
551,363.25 \pm 781,230.21 \\
9088.66 \pm 16,703.74 \\
43.52 \pm 35.34 \\
4982.94 \pm 8010.17 \\
410,828.33 \pm 579,105.69 \\
\text { nd } \\
1,089,885.59 \pm 1,431,076.54\end{array}$ & $\begin{array}{l}\mid 7.55 \pm 8.57 \# \\
47,975.07 \pm 23,504.38 \\
83.89 \pm 20.95 \\
\text { nd } \\
\text { nd } \\
\text { nd } \\
27,26|.33 \pm| 3,562.97 \\
55,402.7 \pm 27,290.26 \\
30 \mid 9.39 \pm 3503.77 \\
|707.85 \pm| 395.20 \\
30.54 \pm \mid 4.32 \\
797.5 \mid \pm 655.34 \\
108,390.72 \pm|| 5,860.37 \\
\text { nd } \\
255,6|9.86 \pm| 76,202.38\end{array}$ \\
\hline
\end{tabular}

Notes: $* P<0.05, \mathrm{NC}$ group versus $\mathrm{HF}$ group. $\# P<0.05$, HF group versus $\mathrm{FI}$ group.

Abbreviations: nd, not detectable; CA, cholic acid; CDCA, chenodeoxycholic acid; DCA, deoxycholic acid; LCA, lithocholic acid; MCA, muricholic acid; UDCA, ursodeoxycholic acid; $\alpha$ MCA, $\alpha$-muricholic acid; $\beta$ MCA, $\beta$-muricholic acid; TCA, tauro-cholic acid; TCDCA, tauro-chenodeoxycholic acid; TLCA, tauro-lithocholic acid; TUDCA, tauro-ursodeoxycholic acid; T $\alpha$ MCA, tauro- $\alpha$-muricholic acid; T $\beta$ MCA, tauro- $\beta$-muricholic acid; BA, bile acid. 


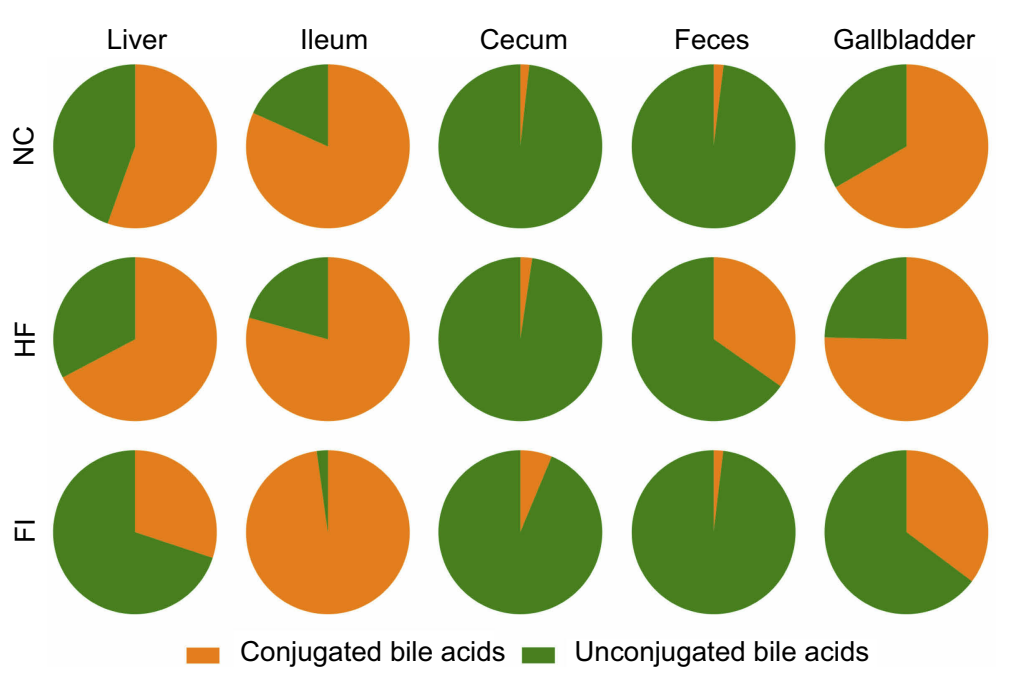

Figure S3 Pie charts of conjugated bile acids and unconjugated bile acids in liver, ileum, cecum, feces and gallbladder. 

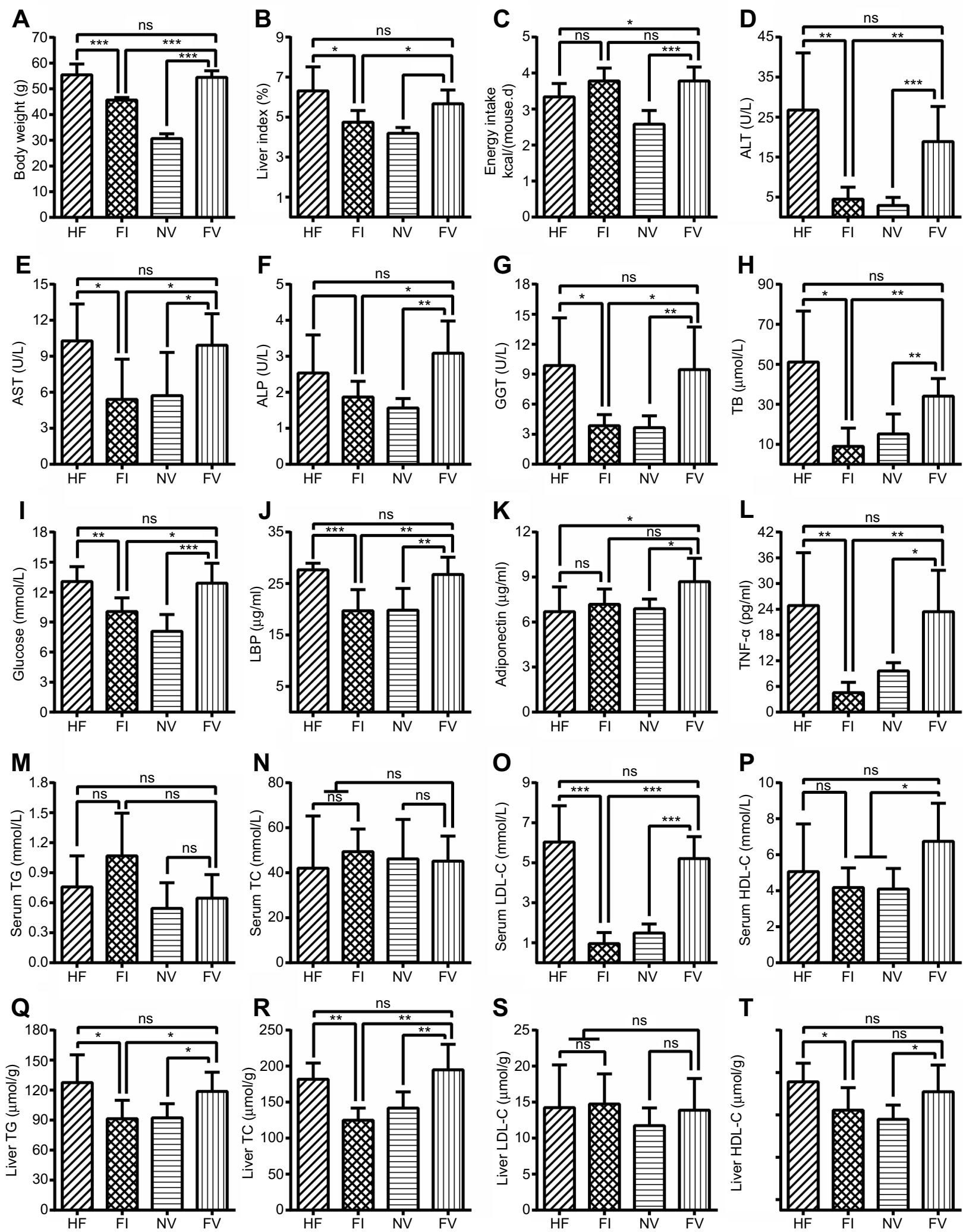

Figure S4 Obesity portraits, serum biochemical indicators and liver lipids in the four groups. (A) Body weight in the 24th week. (B) Liver index. (C) Energy intake in the 24th week. (D) Serum ALT, (E) AST, (F) ALP, (G) GGT, (H) TB, (I) glucose, (J) LBP, (K) adiponectin and (L) TNF-a. (M) Serum triglyceride, (N) total cholesterol, (O) LDLC and (P) HDL-C. (Q) Liver triglycerides, (R) cholesterol, (S) LDL-C and (T) HDL-C. *P<0.05; **P<0.0I; ***P<0.00I; ns, not statistically significant.

Abbreviations: ALT, alanine aminotransferase; AST, aspartate aminotransferase; ALP, alkaline phosphatase; GGT, gamma-glutamyl transpeptidase; TB, total bilirubin; LBP, lipopolysaccharide-binding protein; TNF- $\alpha$, tumor necrosis factor-alpha; TG, triglyceride; TC, total cholesterol; LDL-C, low-density lipoprotein-cholesterol; HDL-C, highdensity lipoprotein-cholesterol. 

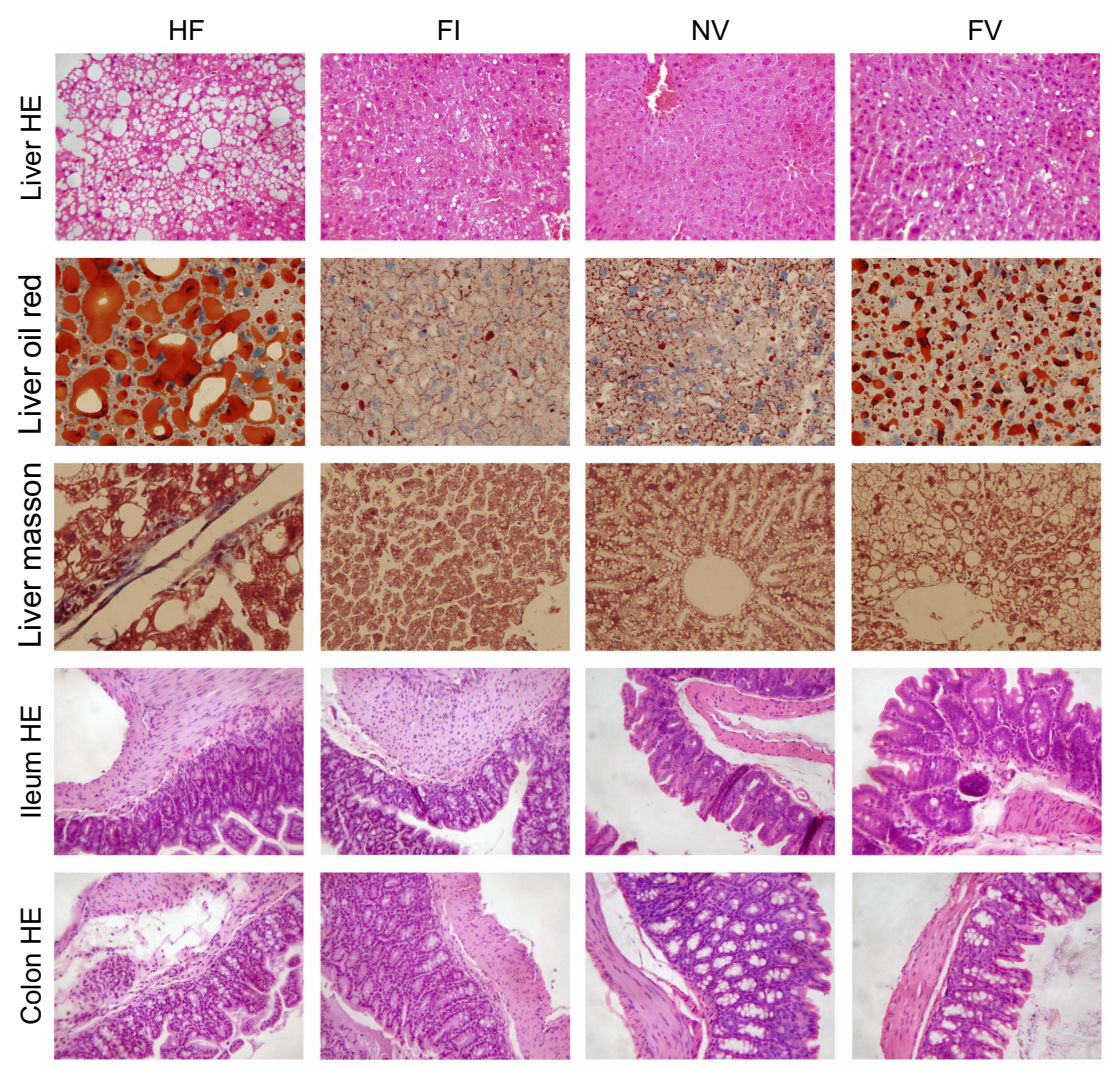

Figure S5 Histopathological staining of liver, ileum and colon tissues in the four groups. Representative liver HE, oil red O staining and Masson's trichrome staining, and HE staining of ileum and colon tissues (200×magnification).

Abbreviations: $\mathrm{HE}$, hematoxylin-eosin; NV, group fed with the control diet and treated with obeticholic acid $(30 \mathrm{mg} /(\mathrm{kg} \cdot \mathrm{d}))$ and a combination of antibiotics; FV, group fed with high fat diet and treated with obeticholic acid $(30 \mathrm{mg} /(\mathrm{kg} \cdot \mathrm{d}))$ and a combination of antibiotics.

\section{Publish your work in this journal}

Drug Design, Development and Therapy is an international, peerreviewed open-access journal that spans the spectrum of drug design and development through to clinical applications. Clinical outcomes, patient safety, and programs for the development and effective, safe, and sustained use of medicines are a feature of the journal, which has also been accepted for indexing on PubMed Central. The manuscript management system is completely online and includes a very quick and fair peer-review system, which is all easy to use. Visit http://www. dovepress.com/testimonials.php to read real quotes from published authors. 